\title{
Experimental classification of quenched quantum walks by dynamical Chern number
}

\author{
Xiao-Ye Xu, Qin-Qin Wang, Si-Jing Tao, Wei-Wei Pan, Zhe Chen, Munsif Jan, Yong-Tao Zhan, Kai Sun, Jin-Shi Xu, \\ Yong-Jian Han, ${ }^{*}$ Chuan-Feng Li $\odot{ }^{\dagger}{ }^{\dagger}$ and Guang-Can Guo \\ CAS Key Laboratory of Quantum Information, University of Science and Technology of China, Hefei 230026, People's Republic of China \\ and CAS Center For Excellence in Quantum Information and Quantum Physics, University of Science and Technology of China, \\ Hefei 230026, People's Republic of China
}

(Received 28 January 2019; published 22 October 2019)

\begin{abstract}
Topology has rapidly become one of the central topics in modern physics because of its ability to explain various interesting phenomena, especially in condensed matter physics. Topological invariants, serving as indicators for different topological phases, have been widely studied in various quantum systems. Generally, topological invariants are defined in (quasi)equilibrium systems through their ground-state manifold and are used to classify different topological phases. Recently, topological invariants in quantum systems far from equilibrium have been taken into account theoretically in quite different ways. Here, the dynamical Chern number, originally introduced in quenches of static systems, is extended to the quenches of periodically driven systems. Moreover, experimental measurements of dynamical topological invariants appearing in different quantum quenches are reported. The results show that the dynamical Chern number offers an intrinsic way to classify quenched quantum walks, and they demonstrate further its relation to quasiequilibrium topological bulk invariants associated with quenched quantum walks between different topological phases. The classifications by the dynamical Chern number are also compared with the classifications by the behavior of the dynamical topological order parameter. The platform used in this study provides an ideal way to investigate the topology in nonequilibrium quantum systems.
\end{abstract}

DOI: 10.1103/PhysRevResearch.1.033039

\section{INTRODUCTION}

The topological properties of various quantum systems have been widely investigated in recent decades [1-9]. At equilibrium, the topological invariants of a system with some symmetries can be explicitly defined on the ground-state manifold of a Hamiltonian [10,11]. For example, the bulk Chern number can be defined in a Chern insulator as the number of edge states through "the bulk-edge correspondence" [12-14]. In addition, the topological invariants provide a natural classification of the topological phases of a system. However, studies about the topological invariants and the intrinsic classification of nonequilibrium processes are scarce, and were only recently started in theory $[15,16]$.

The quench of a quantum system is typically a nonequilibrium process [17-19]. In a standard quantum quench of a static system, the system is initially prepared in the ground state of a Hamiltonian $H_{i}$. Then, its Hamiltonian is suddenly changed to $H_{f}$, and the system evolves under the new Hamiltonian $H_{f}$. Since the topological invariants of a quantum state cannot be changed under unitary evolution,

\footnotetext{
*smhan@ustc.edu.cn

$\dagger$ cfli@ustc.edu.cn
}

Published by the American Physical Society under the terms of the Creative Commons Attribution 4.0 International license. Further distribution of this work must maintain attribution to the author(s) and the published article's title, journal citation, and DOI. nontrivial topological phenomena do not appear during the quantum quench. However, recent investigations have shown that various interesting topological properties emerge during the quantum quench [20-24], e.g., a dynamical topological invariant (dynamical Chern number) can be defined on the momentum-time manifold in the quench of a one-dimensional (1D) two-band quantum system [25]. Interestingly, it has been found that the dynamical Chern number is related closely to the underlying topological invariants of the initial and final Hamiltonians, $H_{i}$ and $H_{f}$, respectively.

Driven systems, especially periodically driven Floquet systems, are also typical nonequilibrium quantum systems. The topological phenomena in driven systems are found to be richer than those in their nondriven counterparts; some topological phenomena can only be observed in driven systems [26-34]. The quasiequilibrium topological properties of a periodically driven system can be explored by the topology of its underlying Floquet Hamiltonian. Quenches of a driven Floquet system are genuine dynamical processes beyond its quasiequilibrium picture.

Quantum walk (QW) [35], which shows distinct phenomena compared to its classical counterparts, has been found useful in quantum algorithms [36] and quantum computation [37,38]. As it contains a natural coupling between the spin and the walker's position, which can be described via Floquet theory originally formulated for periodically driven systems [26], QW has also been shown to be a powerful participant in quantum simulation $[39,40]$. While QW can been implemented in various physical systems [41,42], photonic implementation shows its particular advantages: working in room 
temperature, long coherence time, and high operation fidelity [43]. There have been developed a variety of architectures for photonic QWs [44-49]; however, our recently developed platform [50,51] does have particular advantages: It is free of extra loss and maintains the scalability and stability of conventional time multiplexing protocol; as a result, it can work with a genuine single-photon source. Besides, we can reconstruct the full wave function of the walker at any time of steps and initialize the system to the ground state of any given Hamiltonian via extra adiabatic time evolution. As a consequence, our photonic QW platform provides a powerful tool for investigating intrinsically topological phenomena in a quenched periodically driven system [22].

In a quenched QW, the system is experimentally initialized to the proposition state of the lowest band of the initial Floquet operator $U_{i}$ (and its corresponding Floquet Hamiltonian). Then, the Floquet operator quenches to $U_{f}$ (the Floquet operator can be quenched by system parameters or by time frames) and the system evolves under the new operator. Here, we measure the dynamical Chern number in different quantum quenches and find that it can offer an intrinsic way to classify quenched QWs. In addition, it is found that the dynamical Chern number of a quantum quench is related closely to its underlying quasiequilibrium topology. Furthermore, the dynamical quantum phase transition (DQPT) [52], which is characterized by the zeros of the Loschmidt amplitude and can be used to classify the quenches through the dynamical topological order parameter (DTOP) [20], corresponds to the big circle on the momentum-time manifold. The classifications by the dynamical Chern number and by the number of DQPTs are compared, and we show that they offer different information about the quenched dynamics.

\section{QUASIEQUILIBRIUM TOPOLOGY IN QW}

To reveal the topology hidden in 1D QW, a scheme named "split step" has been proposed [39], where for one full step the time evolution operator $U\left(\theta_{1}, \theta_{2}\right)$ (Floquet operator) is defined as

$$
U\left(\theta_{1}, \theta_{2}\right)=S_{-} R\left(\theta_{2}\right) S_{+} R\left(\theta_{1}\right)
$$

in the standard time frame. $R\left(\theta_{1(2)}\right)$ represents the coin tossing, i.e., rotation of the spin. $S_{ \pm}:=\sum_{x}|x \pm 1\rangle\langle x| \otimes$ $| \pm\rangle\langle \pm|+| x\rangle\langle x|\otimes| \mp\rangle\langle\mp|$ with $|+\rangle=|\uparrow\rangle$ and $|-\rangle=|\downarrow\rangle$ are the bases of the spin, and $x \in \mathcal{Z}$ denotes the index of the lattice site. $S_{ \pm}$are conditional shift operators resulting from the coupling of the spin and the position. The quantum evolution of dynamics until time $t$ (positive integer) can be described by repeating the single-step unitary operator $U$ for $t$ times, given directly by $U^{t}$.

For a periodically driven system with translation invariance, its time evolution operator can be transformed into the quasimomentum space with form $U(t)=$ $\exp \left[-i \int_{0}^{2 \pi} d k H(k) \otimes|k\rangle\langle k| t\right]$, where $k \in[0,2 \pi)$ denotes the quasimomentum in the first Brillouin zone and the effective Hamiltonian $H(k)$ is written as

$$
H(k)=E(k) \hat{\boldsymbol{n}}(k) \cdot \boldsymbol{\sigma} .
$$
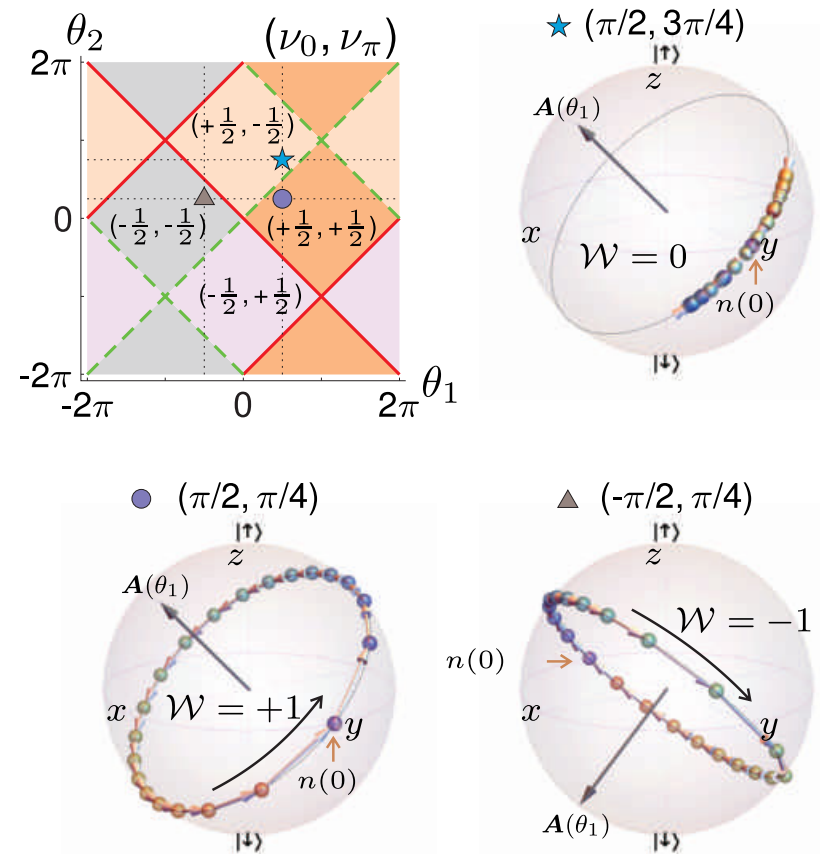

FIG. 1. Complete quasiequilibrium topological phase diagram of the split-step QW, which is given by $\left(v_{0}, v_{\pi}\right)=\left(\frac{v^{\prime}+v^{\prime \prime}}{2}, \frac{v^{\prime}-v^{\prime \prime}}{2}\right)$ with $v^{\prime}$ and $v^{\prime \prime}$ defined as conventional winding numbers on its lower-band manifold in two nonequivalent time frames. The Bloch spheres show the corresponding winding of the system's eigenvectors within the first Brillouin zone in the standard time frame, where the corresponding time evolution operator is given by Eq. (1).

Here, $E(k)$ is the quasienergy dispersion, $\sigma=\left(\sigma_{x}, \sigma_{y}, \sigma_{z}\right)$ is a vector of Pauli matrices, and $\boldsymbol{n} \hat{(k)}$ is a unitary Bloch vector identifying the eigenstate of the coin part [40].

The quasiequilibrium topology hidden in a split-step QW (SSQW) can be defined through its lower-band manifold [the manifold formed by the ground eigenvectors of $H(k), k \in$ $[0,2 \pi)]$. Actually, it has been shown that QWs in 1D and 2D can possess chiral, particle-hole and time-reversal symmetries, and as a consequence can naturally realize all ten classes of nontrivial topological phases $[39,40]$. Bulk topological invariants, i.e., the winding numbers, can be defined in a $1 \mathrm{D}$ QW with chiral symmetry to distinguish topological and trivial phases. By chiral symmetry, we means there is a unitary, Hermitian, and local operator $\Gamma$ that satisfies $\Gamma H \Gamma=-H$ for all of the parameters. In fact, the effective Hamiltonian in Eq. (2) can be used to describe various condensed matter physics, such as the Su-Schrieffer-Heeger model [53], the $p$-wave Kitaev chain [54], and the transverse field Ising model [55]; hence, the topology in such systems can be defined in a similar way. Distinctively, for a periodically driven system, we need to take into account the system's time frames when considering the complete classification of its hidden topology, which is quite different from its static counterpart [29-33]. In our case, there exist two nonequivalent time frames [with the corresponding time evolution operators $U_{1}=\sqrt{R\left(\theta_{1}\right)} S_{-} R\left(\theta_{2}\right) S_{+} \sqrt{R\left(\theta_{1}\right)}$ and $U_{2}=\sqrt{R\left(\theta_{2}\right)} S_{-} R\left(\theta_{1}\right) S_{+} \sqrt{R\left(\theta_{2}\right)}$ in one period]. As shown in Fig. 1, a pair of invariants $\left(v_{0}, v_{\pi}\right)=\left(\frac{v^{\prime}+v^{\prime \prime}}{2}, \frac{v^{\prime}-v^{\prime \prime}}{2}\right)$, with $v^{\prime}$ 
and $v^{\prime \prime}$ defined as conventional winding numbers on its lowerband manifold in two nonequivalent time frames is needed to completely identify the different topological phases [30,31]. The quasiequilibrium topology considered here is defined on the band of the effective Hamiltonian $H(k)$ (taking into account the time frame). It is different from the definition of topology based on the time evolution operator for a periodically driven system, although these two definitions are found to have a close mathematical relationship [26,28,34].

\section{DYNAMICAL TOPOLOGY EMERGENT IN SSQW}

Although, both theoretically and experimentally, the quasiequilibrium topology of the SSQW is understood clearly [29-31,33,39,40,50,56-67], its intrinsic dynamical topology (beyond the quasiequilibrium picture) is still open for further investigation. Recently, the DTOP (an integer-valued number) was introduced to identify different dynamical phases when DQPT happens [20,52]. The behavior of the DTOP was extended to classify the quenches of SSQW and was further experimentally measured in [22]. Here, we use another quantized valued invariant, i.e., the dynamical Chern number [25], to characterize the intrinsic topology hidden in the quenches of SSQW and subsequently classify the quenches. We show that these two classification methods are related closely but are not the same, and that they indicate different aspects of the quenched dynamics.

In a quenched SSQW, the system is initialized to $\left|\psi_{i}\right\rangle=$ $\int_{0}^{2 \pi} f(k) d k\left|\psi_{i}(k)\right\rangle$, where $\left|\psi_{i}(k)\right\rangle$ is the ground state corresponding to the initial Floquet Hamiltonian $H_{i}(k)$ in quasimomentum space and $f(k)$ is the complex amplitude in quasimomentum space that satisfies the normalization condition. Then, the SSQW quenches to another Floquet operator $U_{f}$ corresponding to the effective Hamiltonian $H_{f}(k)$ in quasimomentum space, and the system evolves as $|\psi(k, t)\rangle=$ $e^{-i H_{f}(k) t}\left|\psi_{i}(k)\right\rangle$ for each quasimomentum $k$.

Time evolution, fixed points, and big circles. For convenience, we represent the spin state in its density matrix form. Thus, the spin state with quasimomentum $k$ at time $t$ can be represented as $k$ [25]:

$$
\rho(k, t)=|\psi(k, t)\rangle\langle\psi(k, t)|=\frac{1}{2}\left[1-\hat{\boldsymbol{n}}_{k}(t) \cdot \boldsymbol{\sigma}\right],
$$

and $\hat{\boldsymbol{n}}_{k}(t)$ is the Bloch vector, which is given by

$$
\begin{aligned}
\hat{\boldsymbol{n}}_{k}(t)= & \hat{\boldsymbol{n}}_{k}^{i} \cos \left(2 E_{k}^{f} t\right)+2 \hat{\boldsymbol{n}}_{k}^{f}\left(\hat{\boldsymbol{n}}_{k}^{i} \cdot \hat{\boldsymbol{n}}_{k}^{f}\right) \sin ^{2}\left(E_{k}^{f} t\right) \\
& +\hat{\boldsymbol{n}}_{k}^{i} \times \hat{\boldsymbol{n}}_{k}^{f} \sin \left(2 E_{k}^{f} t\right) .
\end{aligned}
$$

$\hat{\boldsymbol{n}}_{k}^{i}\left(\hat{\boldsymbol{n}}_{k}^{f}\right)$ is the Bloch vector of the spin state determined by the ground state of the initial (final) Hamiltonian $H_{i}(k)$ $\left[H_{f}(k)\right]$ and $E_{k}^{f}$ is the quasienergy dispersion of $H_{f}(k)$ defined in Eq. (2). The dynamics of the Bloch vector $\hat{\boldsymbol{n}}_{k}(t)$ can be interpreted as the winding of the Bloch vector around the axis $\hat{\boldsymbol{n}}_{k}^{f}$ starting from the initial vector $\hat{\boldsymbol{n}}_{k}^{i}$ on the Bloch sphere. Geometrically, the orbit formed by the winding of $\hat{\boldsymbol{n}}_{k}(t)$ is a circle, as shown in Fig. 2. The radius of the circle corresponding to quasimomentum $k$ is written as

$$
r(k)=\sqrt{1-\left(\hat{\boldsymbol{n}}_{k}^{i} \cdot \hat{\boldsymbol{n}}_{k}^{f}\right)^{2}} .
$$

There are two special cases of circles that are extremely interesting. The first case is when the radius of the circle, $r\left(k_{\mathrm{F}}\right)$, is zero, meaning that the corresponding Bloch vector does not change. In this case, the corresponding quasimomentum $k_{\mathrm{F}}$ is called the fixed point of the quench, which plays a key role in constructing the momentum-time manifold in the following. There are two different types of fixed points: one is determined by symmetry; e.g., for a particle-hole symmetry (by particle-hole symmetry, we mean the effective Hamiltonian satisfies $H^{*}=-H$ ), the quasimomenta $k=0$ and $k=\pi$ are always the fixed points, which are independent of the parameters of the quench. The other type is called accidental fixed points, which are dependent on the quench parameters; e.g., accidental fixed points can be found in a quench between two topologically nonequivalent nontrivial phases characterized by $\left(+\frac{1}{2},+\frac{1}{2}\right)$ and $\left(-\frac{1}{2},-\frac{1}{2}\right)$, (see Fig. 3$)$. The second case is when the radius of the circle, $r\left(k_{c}\right)$, is 1 , which means the initial Bloch vector $\hat{\boldsymbol{n}}_{k_{c}}^{i}$ is perpendicular to the final one $\hat{\boldsymbol{n}}_{k_{c}}^{f}$. For the corresponding quasimomentum $k_{c}$, we consider the Loschmidt amplitude of its dynamics [16]:

$$
\operatorname{Tr}\left[\rho\left(k_{c}, t\right) \rho\left(k_{c}, 0\right)\right]=\frac{1}{2}\left[1+\hat{\boldsymbol{n}}_{k_{c}}(t) \cdot \hat{\boldsymbol{n}}_{k_{c}}(0)\right] .
$$

It is clear that the Loschmidt amplitude is zero when the condition $\hat{\boldsymbol{n}}_{k_{c}}(t)=-\hat{\boldsymbol{n}}_{k_{c}}(0)$ is satisfied for some time $t$, indicating the appearance of DQPT [52]. Actually, the state with Bloch vector $-\hat{\boldsymbol{n}}_{k_{c}}(0)$ is always on the big circle due to the symmetry of the big circle. Specifically, at a critical time $t_{c}=T_{k_{c}} / 2$, where $T_{k_{c}}$ is the evolution period for quasimomentum $k_{c}$, the Loschmidt amplitude is $\operatorname{Tr}\left[\rho\left(k_{c}, t\right) \rho\left(k_{c}, 0\right)\right]=0$. As a result, we can establish the direct correspondence between the DQPT (the discontinuity of DTOP for quasimomentum $k_{c}$ ) and the big circle. Therefore, the appearance of the big circle indicates the existence of the DQPT characterized by $k_{c}$ and $t_{c}$, which were experimentally measured in [22].

Figure 2 demonstrates the geometric behaviors of the Bloch vector $\hat{\boldsymbol{n}}_{k}(t)$ in two typical quenches. Figure 2(a) shows the two quench strategies in a phase diagram. Both of the quenched SSQWs start from the eigenstate of a Hamiltonian with flat band in the nontrivial phase (denoted as a circle), but one quenches to a SSQW in the same nontrivial phase (triangle) and the other quenches to a SSQW in the trivial phase (diamond). In Fig. 2(b), we plot the circle's radius $r(k)$ as a function of quasimomentum $k \in[0,2 \pi)$ for the two selected quenches. It shows that there is no big circle (no DQPT) in the former case and two big circles (two different types of DQPT) appear in the latter case. In addition, there are no additional (accidental) fixed points in both of the quenches aside from the fixed points $k=0$ and $\pi$ determined by the particle-hole symmetry. Figure 2(c) shows the dependence of the appearance of the big circle (DQPT) on the quenching strategies. The starting SSQW of the quenches is fixed in the nontrivial phase but the final SSQW of the quench is along a strategy line [see Fig. 2(a)]; hence, we plot the maximal radius along the strategy line in Fig. 2(c). It is clear that the appearance of the big circle (DQPT) and the phase transition occur at the same time.

Momentum-time manifold, Berry curvature, and dynamical Chern number. Besides using the number of the big circles formed during the quench in the range $k \in[0, \pi)$, 
(a)

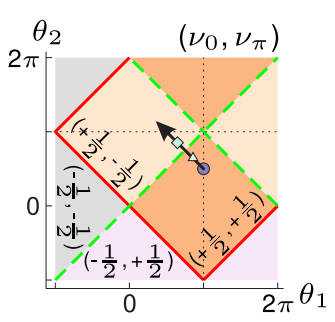

(b)

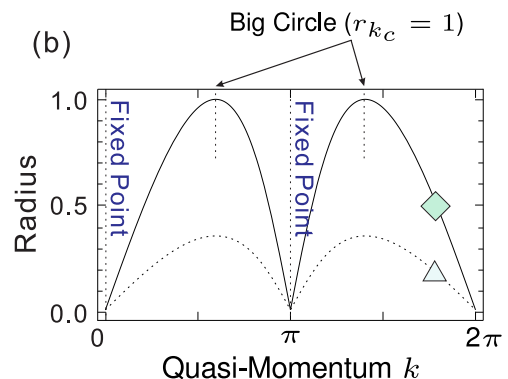

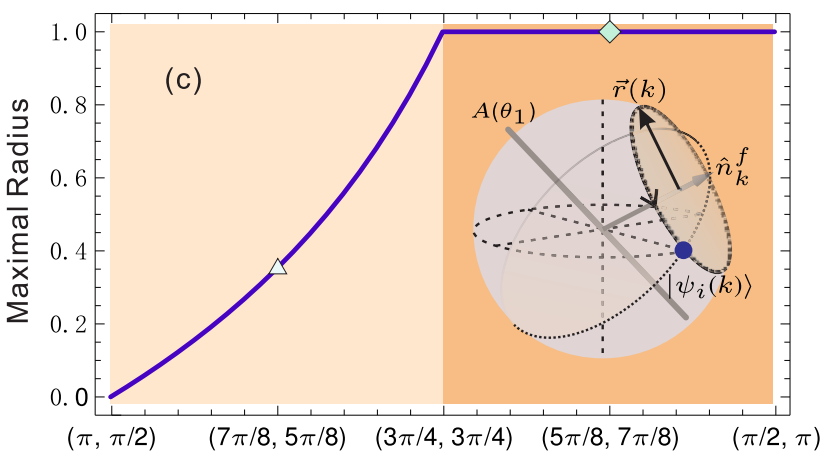
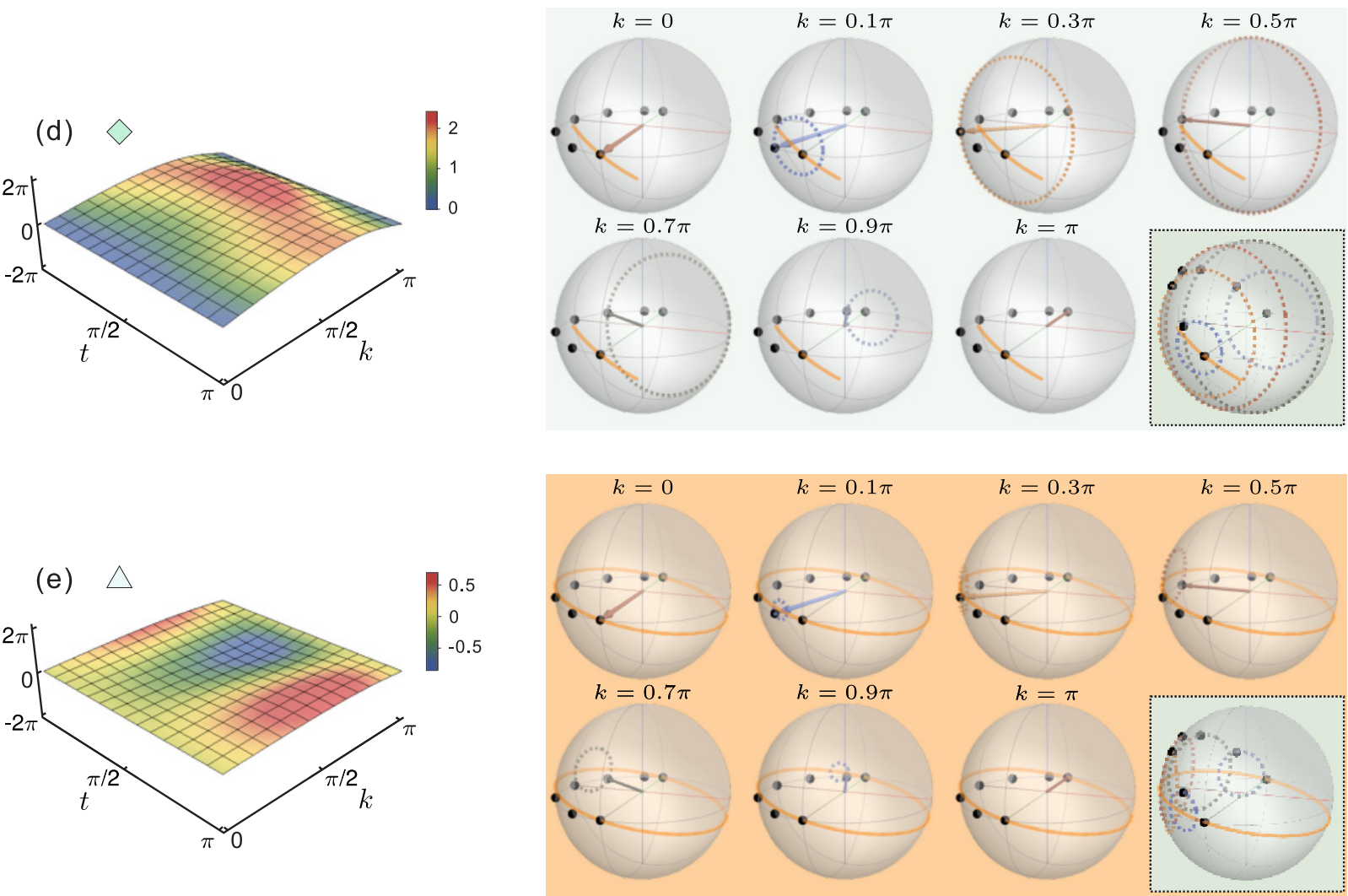

FIG. 2. (a) Quenching strategies in a phase diagram, which start from the flat band in the nontrivial phase $\left(+\frac{1}{2},+\frac{1}{2}\right)$ with $\theta_{1}=\pi$ (circle). The ends are located in a line $\theta_{2}=-\theta_{1}+3 \pi / 2$ (solid arrow). (b) Dependence of the circle's radius on quasimomentum $k$. The solid line (quench ends at $\theta_{1}=5 \pi / 8$ and $\theta_{2}=7 \pi / 8$ ) has two big circles (radius $=1$ ) and the dashed line (quench ends at $\theta_{1}=7 \pi / 8$ and $\theta_{2}=5 \pi / 8$ ) has no big circle in the first Brillouin zone. Two fixed points $(k=0, \pi)$ exist in both cases. (c) Maximum value of the circle's radius for different quenching strategies. The inset shows the evolution of the time evolved state, which forms a black dashed circle on the surface of the Bloch sphere. (d) and (e) Corresponding Berry curvatures in momentum-time manifold (left panel) and time evolution circles for some given quasimomenta (right panel). The associated Chern number is 1 for (d) and 0 for (e). The black points denote the initial state and the arrow shows the Bloch vector for the corresponding momenta. The orange solid circle shows the ground states of the effective final Hamiltonian.

corresponding to the types of discontinuity of DTOP, i.e., the types of DQPT, to classify quenched SSQW [22], the dynamical Chern number, defined as the Chern number on the momentum-time manifold [25], provides another way to classify quenches.

With the periodic character of the dynamics, the topology of the time can be viewed as $S^{1}$ after performing the rescaling $t^{\prime}=\frac{t}{E_{k}^{f}}$. Then, combining this with the $S^{1}$ topology of the 1D Brillouin zone (BZ), the total momentum-time manifold has a topology $S^{2}$. Note that the momentum-time manifold is separated into several submanifolds by the fixed points $k_{\mathrm{F}} \mathrm{S}$. For the fixed point $k_{\mathrm{F}}$, the initial Bloch vector $\hat{\boldsymbol{n}}_{k_{\mathrm{F}}}^{i}$ is parallel or antiparallel to the final Bloch vector $\hat{\boldsymbol{n}}_{k_{\mathrm{F}}}^{f}$ in the quench.

In each momentum-time submanifold, there is a natural mapping to the Bloch vector, i.e., $\hat{\boldsymbol{n}}_{k}(t): S^{2} \rightarrow S^{2}$; then the Chern number can be defined by the Bloch vector as [25]

$$
C_{\mathrm{dyn}}^{m}=\frac{1}{4 \pi} \int_{k_{m}}^{k_{m+1}} d k \int_{0}^{\pi} d t^{\prime}\left(\hat{\boldsymbol{n}} \times \partial_{t^{\prime}} \hat{\boldsymbol{n}}\right) \cdot \partial_{k} \hat{\boldsymbol{n}},
$$



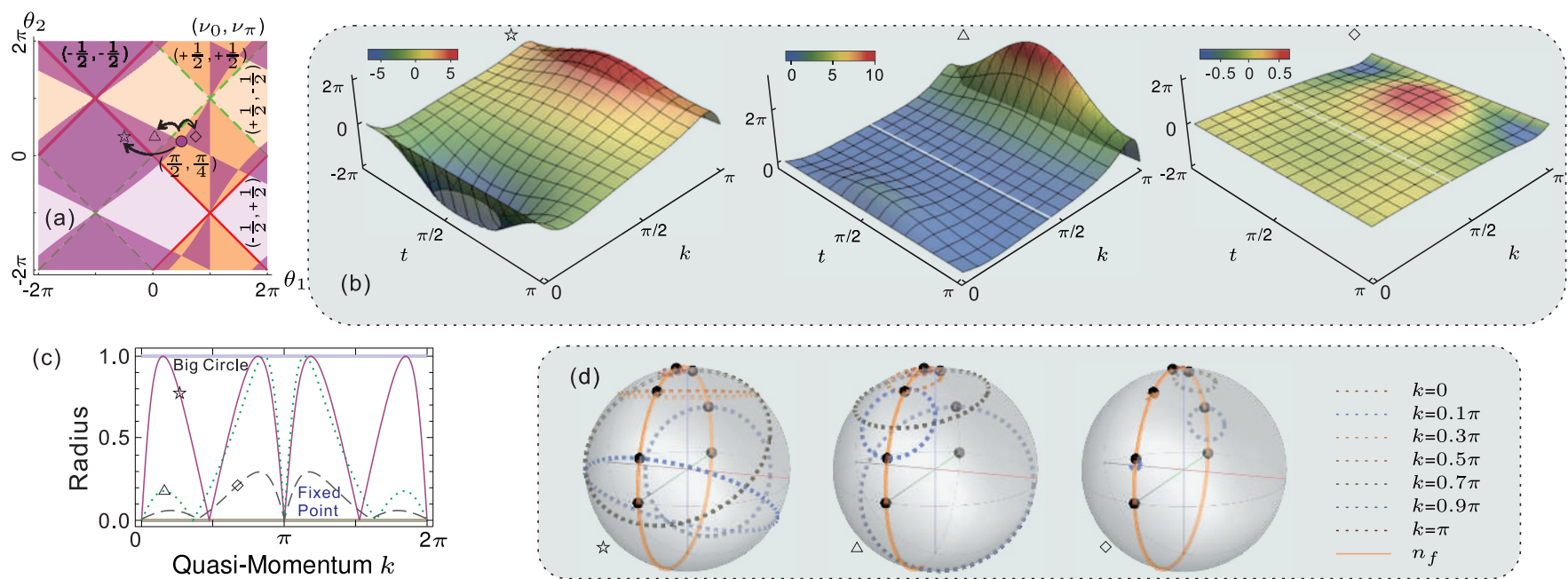

FIG. 3. (a) Three quenching strategies in a phase diagram. All of the quenches start in the eigenstate of the Hamiltonian in the topologically nontrivial phase $\left(+\frac{1}{2},+\frac{1}{2}\right)$ with $\left(\theta_{1}=\pi / 2, \theta_{2}=\pi / 4\right)$ (circle), but they end in three different phases, i.e., in the same topological nontrivial phase with $\left(\theta_{1}=3 \pi / 4, \theta_{2}=\pi / 3\right)$ (diamond), in another nonequivalent topological nontrivial phase $\left(-\frac{1}{2},-\frac{1}{2}\right)$ with $\left(\theta_{1}=-\pi / 2, \theta_{2}=\pi / 3\right)$ (star), and in a trivial phase $\left(+\frac{1}{2},-\frac{1}{2}\right)$ with $\left(\theta_{1}=\pi / 36, \theta_{2}=\pi / 3\right)$ (triangle). The purple shadows show the regions where accidental fixed points appear. (b) Berry curvatures in momentum-time manifold for the three quenches. The dynamical Chern number is 1 for the triangle strategy and 0 for the other two strategies. (c) Dependence of the circle's radius on the quasimomentum $k$ for the three quenching strategies: red solid line for the star strategy, green pointed line for the triangle strategy, and black dashed line for the diamond strategy. All of the three quenches have accidental fixed points in the range $[0, \pi)$ but they have different numbers of big circles, i.e., two for the star strategy, one for the triangle strategy, and zero for the diamond strategy. (d) Corresponding time evolution circles (dashed) for quasi-momenta $k=$ $0,0.1 \pi, 0.3 \pi, 0.5 \pi, 0.7 \pi, 0.9 \pi, \pi$. The solid circle denotes the Bloch vector of the ground state of the final effective Hamiltonian.

where $m$ denotes the $m$ th submanifold, $k_{m}$ is the $m$ th fixed quasimomentum, and $t^{\prime}$ is the rescaled time. The integral kernel is the well-known Berry curvature in the momentum-time submanifold. In Figs. 2(d) and 2(e), we show the theoretical Berry curvatures in two typical quenches: one is between the same nontrivial phases and the other is between the nontrivial and trivial phases. As discussed in Ref. [25], the dynamical Chern numbers defined on the submanifold take an integer value, i.e., 0 (trivial) or \pm 1 (nontrivial). In the quenched SSQW, the whole dynamical Chern number is defined as the sum of the dynamical Chern numbers in each submanifold whose quasimomenta are in $[0, \pi)$, i.e., $C_{\mathrm{dym}}=\sum_{m=1}^{n} C_{\mathrm{dym}}^{m}$, where $k_{n+1}=\pi$, and we use it to classify the quenched SSQW. Following this definition, in the quenching strategies considered in Fig. 2 the dynamical Chern number is 0 for the triangle and 1 for the diamond.

Classification of the quenched SSQW. The whole dynamical Chern number defined on the global time-momentum manifold reads $C_{\mathrm{dyn}}=\frac{1}{2}\left[\left(\hat{\boldsymbol{n}}_{k=0}^{i} \cdot \hat{\boldsymbol{n}}_{k=0}^{f}\right)-\left(\hat{\boldsymbol{n}}_{k=\pi}^{i} \cdot \hat{\boldsymbol{n}}_{k=\pi}^{f}\right)\right]$, where the information about the accidental points can cancel out each other. Therefore, according to the value of $\left|C_{\mathrm{dyn}}\right|$, the quenches can be classified into two classes:

(I) $C_{\mathrm{dym}}=1$ : one big circle appears (indicating only one type of DQPT occurs) when $k$ is between 0 and $\pi$. All the quenches between the trivial and nontrivial phases belong to this class. For the SSQW, the quenches between different time frames also belong to this class. According to the appearance of the accidental fixed point (equal to the dynamical Chern number on the submanifold), this class can be divided further into two subclasses: (1a) There is no accidental fixed point appearing (see the diamond strategy in Fig. 2, the strategy in Fig. 5, or the strategy in Fig. 6). (1b) There is an accidental fixed point appearing and separating the momentum-time manifold into two submanifolds and only one big circle; i.e., only one type of DQPT appears in one of the submanifolds (see triangle strategy in Fig. 3).

(II) $C_{\mathrm{dym}}=0$ : all the quenches, except the quenches between the topologically trivial and nontrivial phases (class I), belong to this class. According to the appearance of the accidental fixed point, this class can be divided further into two subclasses: (0a) no accidental fixed point appears between 0 and $\pi$. In this case, there is no big circle (no DQPT) or there are two big circles, i.e., two different types of DQPT (see the triangle strategy in Fig. 2 or the two examples in Fig. 7). (0b) there is an additional accidental fixed point appearing and separating the momentum-time manifold into two submanifolds between 0 and $\pi$ (see the star strategy in Fig. 3 or the case in Fig. 8).

\section{EXPERIMENTAL RESULTS}

Benefiting from our recently developed large-scale QW platform with the full wave function accessible, we can reconstruct the wave function of the SSQW for each step. The spin states for each quasimomentum $k$ can be obtained further by Fourier transformation of the real wave function [50]. By plotting the Bloch vector of each quasi-momentum $k$ on the Bloch sphere along the steps, we can get the geometrical feature of $\hat{\boldsymbol{n}}_{k}(t)$ during the quench. In particular, by calculating the radius of the fitted circles, we can determine whether there is a big circle or whether there is an additional fixed point. In addition, with the fitted circles, the Berry curvature in the momentum-time manifold can be calculated further, and the corresponding dynamical Chern number can be calculated. 


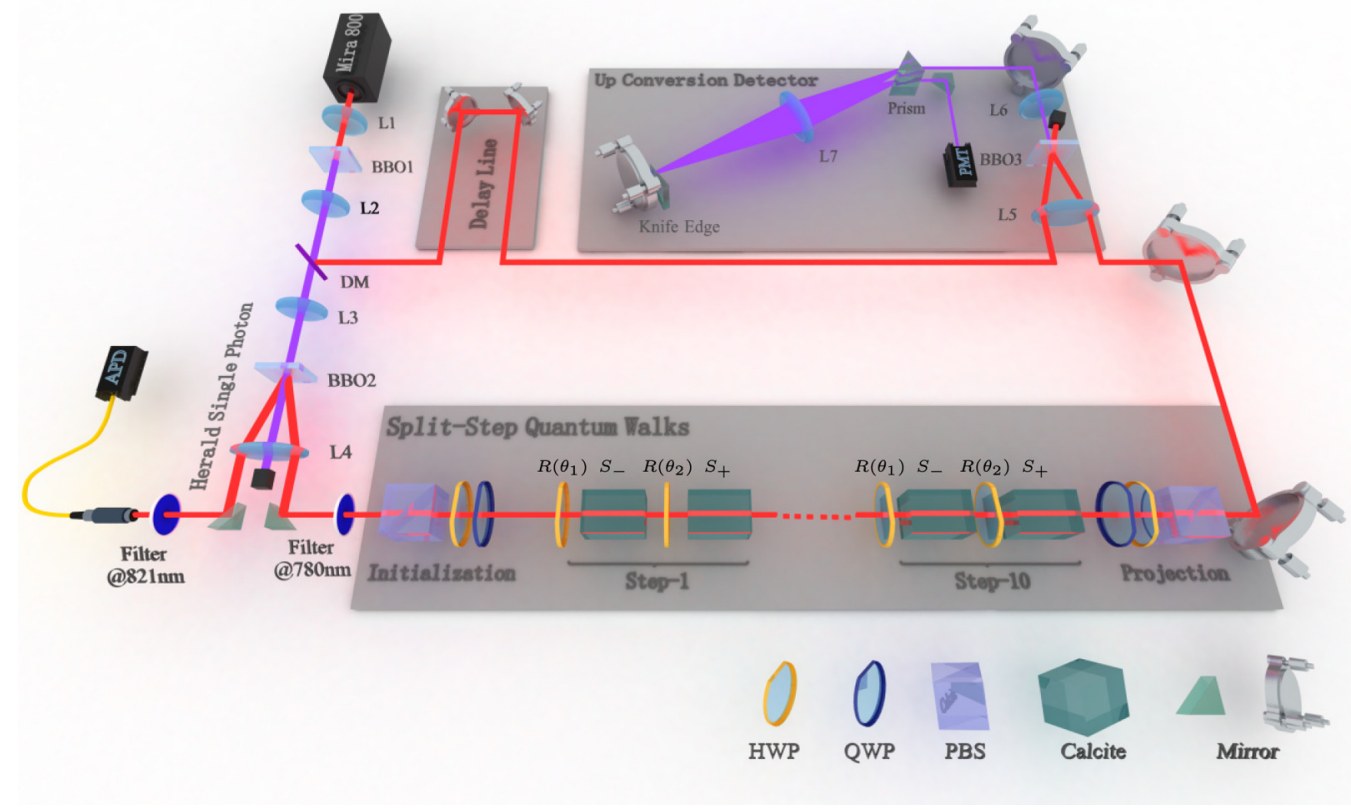

FIG. 4. Sketch of the experimental setup. A pulsed laser with center wavelength $800 \mathrm{~nm}$, pulse duration $150 \mathrm{fs}$, and repetition rate $76 \mathrm{MHz}$ is focused on the first $\beta-\mathrm{BaB}_{2} \mathrm{O}_{4}$ crystal (BBO1). A second harmonic at $400 \mathrm{~nm}$ is generated and used to pump $\mathrm{BBO} 2$ for generating the herald single photon in a beamlike SPDC. The idle photon at $821 \mathrm{~nm}$ is collected and guided to an advanced photon detector (APD) for predicting the signal photon at $780 \mathrm{~nm}$, which is collimated and guided to the quantum walks module. The residual laser in the second harmonic generation is separated from the double frequency laser by a piece of dichroic mirror and delayed as the pumper in our homemade up-conversion detector. The quantum walks module contains three parts: polarization initialization, quantum walks, polarization projection. A full step of quantum walk contains two split steps with one half-wave plate (HWP) and one calcite crystal in each for implementing $R\left(\theta_{1}\right) / R\left(\theta_{2}\right)$ and $S_{-} / S_{+}$respectively. Each piece of calcite is cut collinearly and, with a designed length $8.98 \mathrm{~mm}$, can realize a 5 ps time delay for vertical wave packets. The signal photon after the polarization analyzer is guided to the up-conversion detector, which contains a $4 f$ system acting as a spectrum filter for blocking the scattering photons of the strong pumper. The upconversion signal is counted by a photomultiplier tube (PMT). L, lens; QWP, quarter wave plate; PBS, polarized beam splitter.

The number of big circles (corresponding to different types of DQPT and the number of discontinuities of DTOP) and the value of the dynamical Chern number can be compared. By experimentally obtaining these quantities, we can classify the quenches of SSQW into different classes.

Experimental setup. The experiments are performed on our recently developed novel QW platform with genuine single photons [50,51], whose sketch is shown in Fig. 4. A heralded single photon generated in a spontaneous parametric down conversion (SPDC) is adopted as the walker. Its polarization acts as the spin- $\frac{1}{2}$ coin with the correspondence $|H\rangle \leftrightarrow$ $\left|\uparrow_{z}\right\rangle$ and $|V\rangle \leftrightarrow\left|\downarrow_{z}\right\rangle$, with $|H\rangle$ representing the horizontal polarization and $|V\rangle$ representing the vertical polarization. The lattice is composed of time bins with a time interval of 5 ps. Coin tossing is implemented with a half-wave plate (HWP). The spin-orbit coupling is realized with a birefringent crystal (calcite) cut collinearly with a length $8.98 \mathrm{~mm}$. A polarizer is used to analyze the walker's spinor state. After passing through the polarizer, the photon is counted by an up-conversion single photon detector, which can obtain its arrival time with a precision of less than a picosecond. By using an interference method, we reconstruct the final wave function via maximal likelihood estimation. The associated wave function in momentum representation is obtained by performing Fourier transformation on the reconstructed wave function in temporal space.
System initialization in our SSQW. In a quenched SSQW, we initially prepare the system in the proposition state of the lower band of the initially effective Floquet Hamiltonian $H_{i}$. For convenience, we always begin the preparation process from a Hamiltonian with a flat band. If the initial SSQW of the quench is in the trivial phase, first we can prepare the system in the state $\left|\downarrow_{y}\right\rangle|0\rangle$, indicating that the walker is at the origin having a spin pointing toward the $y$ direction. This state corresponds to the ground state of the flat-band Floquet Hamiltonian with parameter $\theta_{2}=\pi$. With some further adiabatic steps of SSQWs, we can transfer the state to a ground state of any given target Hamiltonian in the trivial phase.

It is a bit more complicated to prepare the ground state in the nontrivial phase. First, we initialize the system in the state $|x=0 \uparrow\rangle$. Then, we perform a full-step QW with parameters $(\pi, \pi / 2)$. Finally, we perform an additional spin rotation along the $\sigma_{z}$ axis with an angle $\pi / 2$. Consequently, the system is prepared in the state $(|-1 \uparrow\rangle-i|0 \downarrow\rangle) / \sqrt{2}$, corresponding to a superposition state of the lower band of the nontrivial flat-band Floquet Hamiltonian with parameter $\theta_{1}=\pi$. Starting from this state, we can prepare the ground state of any target Floquet Hamiltonian in the same nontrivial phase by performing additional adiabatic SSQWs. Note that adiabatic transfer is always valid due to the gap existence in Floquet Hamiltonians. 
(a)

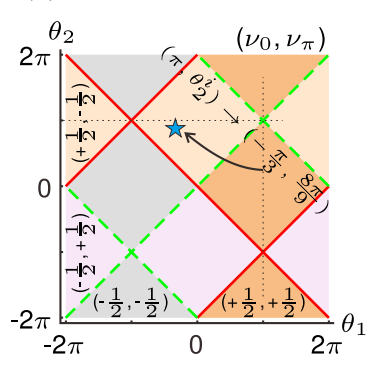

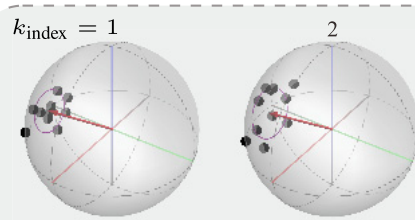

$k_{\text {index }}=7$

(b)

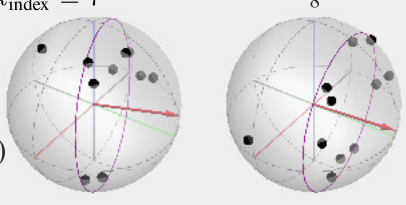

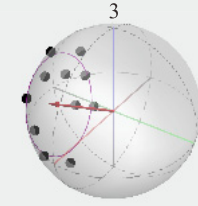

9

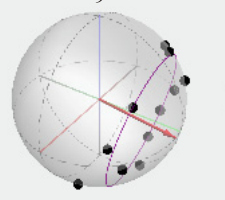

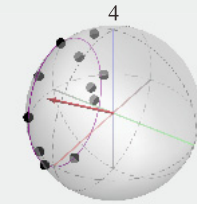

10

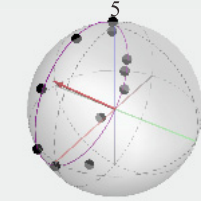

11

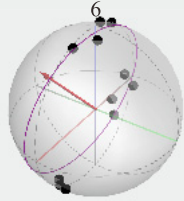

12

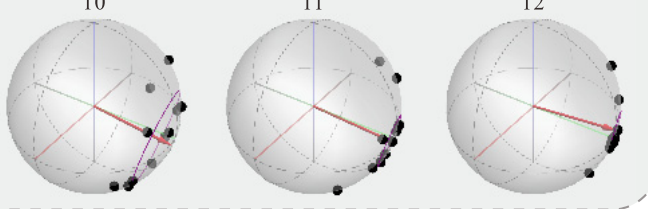

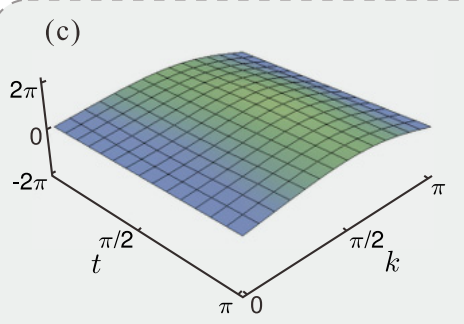
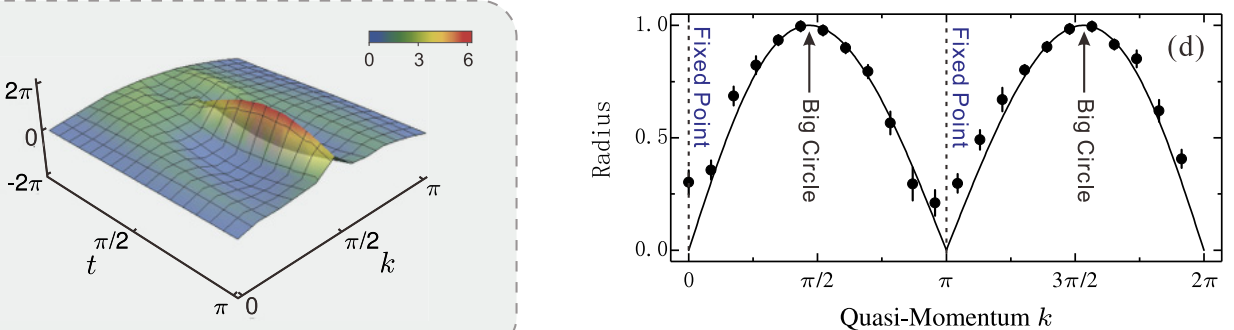

FIG. 5. Experimental results for a quantum quench between topologically nontrivial and trivial phases. (a) Quenching strategy in a phase diagram. The quench starts from the flat band in phase $\left(+\frac{1}{2},+\frac{1}{2}\right)$ with $\theta_{1}=\pi$ and ends it in phase $\left(+\frac{1}{2},-\frac{1}{2}\right)$ with $\theta_{1}=-\pi / 3, \theta_{2}=8 \pi / 9$. (b) Time evolution of the Bloch vectors for different quasimomenta $k$ (indexed by the number on the top of each Bloch sphere) shown as black points. The circles on the sphere are fitted from the states, indicating the time evolution trajectories. The red vectors indicate the direction of the Bloch vector of the effective final Hamiltonian. (c) Corresponding Berry curvature in the momentum-time manifold, where the left panel is for the theory and the right panel is for the experiment. (d) Radius of the time evolution circle as a function of quasimomentum $k$. The points denote experimental results and the solid line denotes the theoretical prediction. The error bars represent standard errors.

After preparing the ground state of the initial SSQW, the system is suddenly changed to another SSQW by modifying the parameters of the SSQW or changing the time frames. In all of the study cases in this work, the total number of quantum quenches is set to 10 . It should be noted here that the total number of site occupations of the walker, which subsequently gives the sampling size $\mathcal{N}$ in quasimomentum space, is determined not only by the total number of steps in a quantum quench but also the number of site occupations of the system's initial state.

Experimental analysis of the types of DQPT and the $d y$ namical Chern number. For each evolution step $t$ after the quench, we can reconstruct the full wave function $\Psi(t)$ via interference measurement and quantum tomography [50]. Then, the time evolved state $|\psi(k, t)\rangle$ for each quasimomentum $k$ can be obtained by performing Fourier transformation on the reconstructed wave function. Then, we can plot the states $|\psi(k, t)\rangle$ for different steps on a Bloch sphere. To determine the time evolution circle for each quasimomentum $k$, first we determine the optimal plane where the circle is located, by fitting the experimental results with a linear regression model. Then, the circle is determined by the intersection of the optimal plane and the Bloch sphere, from which the center and the radius of the circle can also be obtained. By plotting the radius of the circle along the quasimomentum, we can determine the fixed momentum $k_{\mathrm{F}}$ in the quench. In Figs. 5, 6, and 8, we demonstrate this process for different quenches, where panel (b) shows the fitted time evolution circles and panel (d) shows the circle radius for varying quasimomentum $k$ for all of the cases. Here in panel (b) the quasimomentum for each Bloch sphere is given by the index of sampling, $k_{\text {index }}$, within the first Brillouin zone $k \in[0,2 \pi]$, and in term $k_{\text {index }}$ the corresponding quasimomentum reads $k=\frac{2 \pi}{\mathcal{N}-1}\left(k_{\text {index }}-1\right)$, where the sampling size $\mathcal{N}$ equals 24 for Fig. 5 or 26 for Figs. 6 and 8. For the quasimomenta near the fixed points, whose time evolution circles have radii near zero, the systematic errors result in the experimental data diverging from their theoretical predictions more significantly than the other points. However, these noises are local and consequently do not affect the final reading out of the dynamical Chern number.

To form the momentum-time manifold and define the dynamical Chern number, the time should be rescaled for each quasimomentum according to the associated oscillation frequency determined by the eigenenergy $E_{k}^{f}$. The eigenenergy $E_{k}^{f}$ can also be extracted from the reconstructed full wave function of a series of consecutive steps if the following two requirements are satisfied: (1) the total number of steps should be large enough to at least cover one oscillation period and (2) the oscillation period (the minimal period appearing at $k=$ $0, \pi$ that is determined by the gap) should be much larger than 1 since the minimal timescale is 1 in the QW. Here, we obtain the oscillation period by fitting the Loschmidt amplitude $\langle\psi(k, 0) \mid \psi(k, t)\rangle$ for each quasimomentum $k$, where $|\psi(k, 0)\rangle$ is the initial state of quasimomentum $k$ [22]. With the experimentally determined oscillation period, we can rescale the time and construct the momentum-time manifold.

The Berry curvature in the quasimomentum manifold is defined in Eq. (7) for a continuous scenario. In our experiment, the time $t$ is discrete and is not regular, modulo $2 \pi$, 
(a)

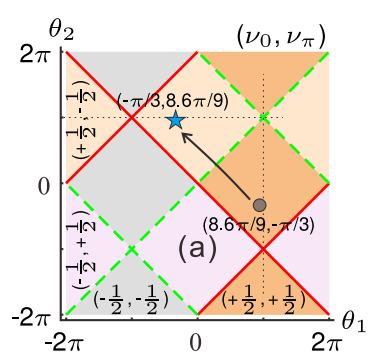

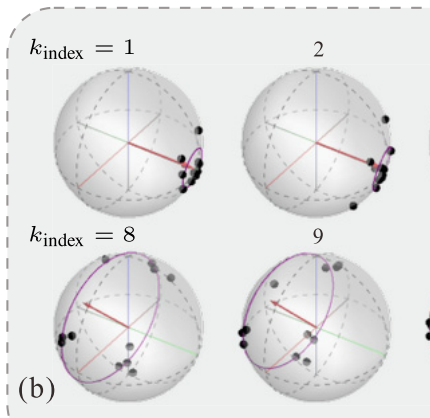

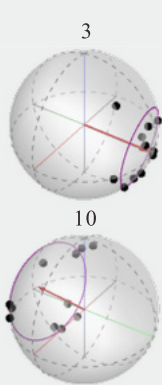

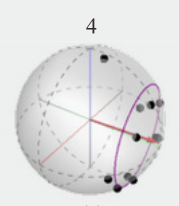

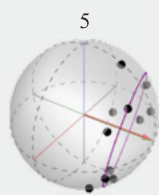

12

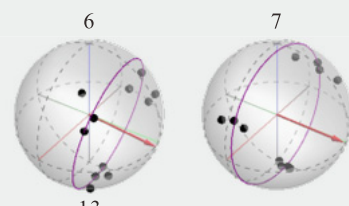

13
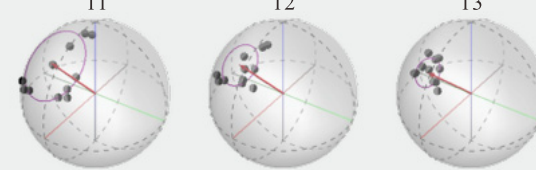

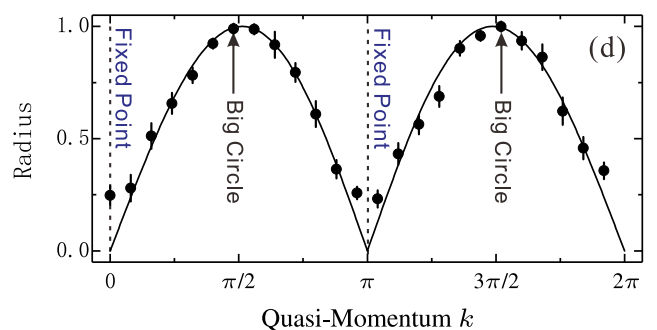

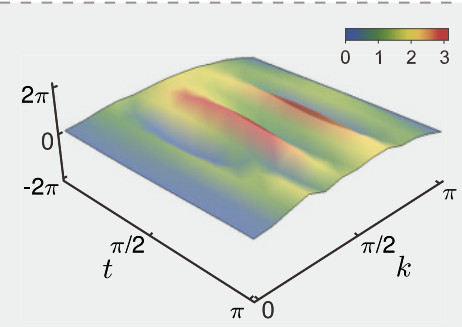

Quasi-Momentum $k$

FIG. 6. Experimental results for a quantum quench driven by suddenly changing the time frames to a nonequivalent one. In this scenario, the system is initialized in the ground state of a Hamiltonian in a topologically nontrivial phase $\left(+\frac{1}{2},+\frac{1}{2}\right)$ with $\left(\theta_{1}=8.6 \pi / 9, \theta_{2}=-\pi / 3\right)$ and is then evolved after suddenly changing its time frame, which is equivalent to changing the system's parameters to $\left(\theta_{1}=-\pi / 3, \theta_{2}=8.6 \pi / 9\right)$. (a) Quenching strategy in a phase diagram. (b) Time evolution of the Bloch vectors for different quasimomenta $k$ (indexed by the number on the top of each Bloch sphere) shown as points. The circles on the sphere are fitted from the states, indicating the time evolution trajectories. The red vectors indicate the direction of the eigenstate of the effective final Hamiltonian. (c) Corresponding Berry curvature in the momentum-time manifold, where the left panel is for theory and the right panel is for the experiment. (d) Radius of the time evolution circle as a function of quasimomentum $k$. The points note the experimental results and the solid line denotes the theoretical prediction. The error bars represent the standard errors.

after the rescaling. To overcome this problem, we use the time evolution circles obtained on the Bloch sphere from our experimental data to make the time $t$ continuous. Then, the Berry curvature and the dynamical Chern number are calculated using Eq. (7). With the value of the dynamical Chern number on each submanifold, the quenches of SSQW can be classified further.

Classification of the quenches of SSQW by dynamical Chern number. Now, we demonstrate the behavior of the quenches in class (I). First, we consider a quench, starting from a flat band SSQW in the nontrivial phase $\left(+\frac{1}{2},+\frac{1}{2}\right)$ with $\theta_{1}=\pi$ and ending in the trivial phase $\left(+\frac{1}{2},-\frac{1}{2}\right)$ with $\theta_{1}=-\pi / 3, \theta_{2}=8 \pi / 9$. Figure 5 shows the experimental results. The time evolution circles of the spin on the Bloch sphere are shown in (b), and the radii of the fitted circles, $r(k)$, are shown in (d). It is clear that there is one big circle $\left[r\left(k_{c}\right)=1\right.$ at $k_{c}=1.764$, which is obtained from the theoretical calculations based on the theory of DQPT] and there are two fixed momenta $k=0$ and $\pi$. The Berry curvature in the momentum-time submanifold is given in the right panel of (c) with its theoretical expectation in the left panel. The dynamical Chern number, which is calculated from the Berry curvature obtained from our experimental data, is 1.059 , which is consistent with the theoretical prediction of 1. As mentioned above, the appearance of the big circle is related closely to the discontinuity of the DTOP $[20,22]$ and the Loschmidt amplitude $\left\langle\psi\left(k_{c}, 0\right) \mid \psi\left(k_{c}, t_{c}\right)\right\rangle$ becomes 0 at some odd time of $t_{c}$ (here the critical time $t_{c}$ is 1.443 , which is obtained from theoretical calculations), where $k_{c}$ is the critical quasimomentum related to the big circle. The behavior is consistent with our previous results, and it can also be mapped to some DQPT in corresponding many-body systems [22,52].

We consider further the peculiar quench of the periodically driven systems achieved by suddenly changing the time frame. In such a quench, the parameters $\theta_{1}$ and $\theta_{2}$ remain the same before and after the quench. In the experiment, first we prepare the system in the ground state of a Floquet Hamiltonian in the topological phase $\left(+\frac{1}{2},+\frac{1}{2}\right)$ with parameters $\theta_{1}=8.6 \pi / 9$, $\theta_{2}=-\pi / 3$ by adopting the adiabatic method. Then, without changing the system's parameters, the system's time frame is suddenly changed to a nonequivalent one. In the final time frame, the Floquet Hamiltonian with the same parameters is actually located in the trivial phase. Therefore, results similar to the previous quench are expected. Figure 6 shows the experimental results. The time evolution circles on the Bloch sphere are shown in (b) and the radius of the circles are shown in (d). From the reconstructed Berry curvature in (c), we can obtain the dynamical Chern number, which is found to be 1.127. It is clear that these two quantum quenches demonstrate similar dynamical behavior. In addition, the dynamical Chern number indicates that they are in the same class.

Finally, we demonstrate the behavior of the quenches in class (II). We experimentally investigate the quenches between the SSQWs in the same phases. As an example, we initially prepare the system in the ground state of the Floquet Hamiltonian corresponding to the SSQW in the phase $\left(+\frac{1}{2},+\frac{1}{2}\right)$ with parameters $\theta_{1}=\pi / 3, \theta_{2}=2 \pi / 3$. Then, 

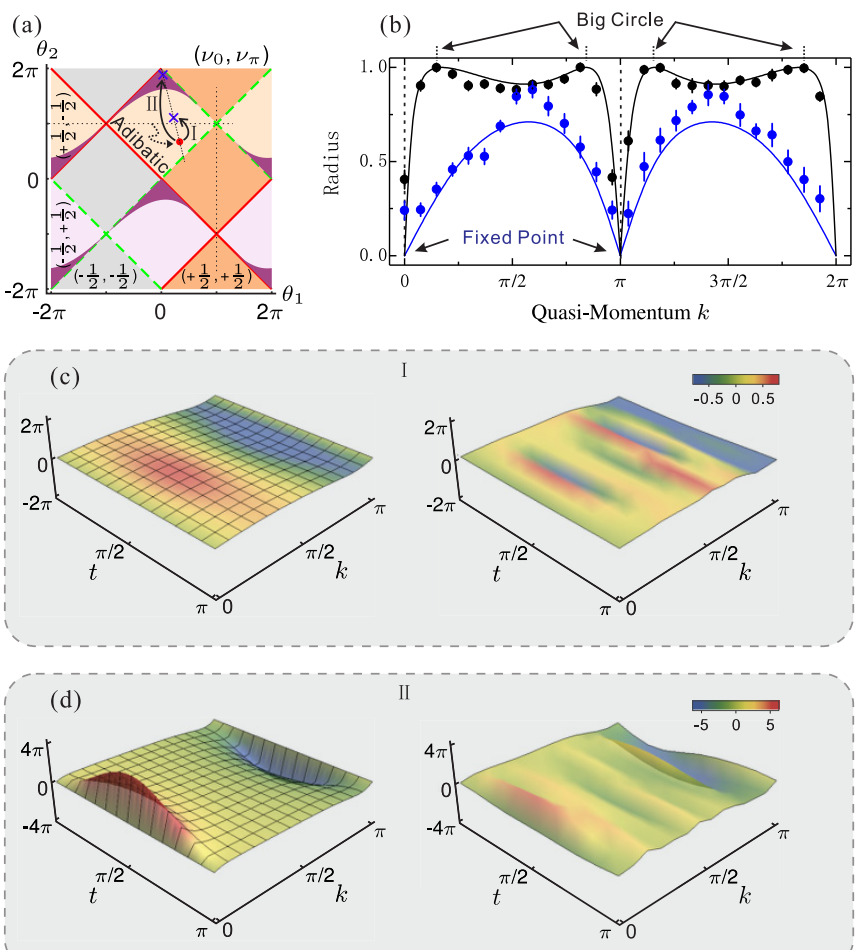

FIG. 7. Experimental results for quantum quenches inside the same trivial phases. (a) Quenching strategies in a phase diagram. We start the quenches from a given Hamiltonian in the trivial phase $\left(+\frac{1}{2},-\frac{1}{2}\right)$ with $\theta_{1}=\pi / 3, \theta_{2}=2 \pi / 3$. To initialize the system, three-step adiabatic SSQWs are adopted from the ground state in the flat band. The quench ends in strategy (I) with $\theta_{1}=2 \pi / 9, \theta_{2}=$ $10 \pi / 9$ or in strategy (II) with $\theta_{1}=\pi / 36, \theta_{2}=17 \pi / 9$. The purple regions show where the two big circles appear. (b) Radius of the time evolution circle as a function of quasimomentum $k$. The blue and black points denote experimental results for strategy (I) and strategy (II), respectively, and the solid lines denote their corresponding theoretical predictions. The error bars represent standard errors. (c) and (d) Relevant Berry curvatures in the momentum-time manifold, where the left panel is for the theory and the right is for the experiment.

the SSQW is suddenly changed to the SSQW in the same phase with parameters $\theta_{1}=2 \pi / 9, \theta_{2}=10 \pi / 9$ and $\theta_{1}=$ $\pi / 36, \theta_{2}=17 \pi / 9$ [the quenching strategies are shown in Fig. 7(a). Figures 7 shows the experimental results. It is shown that there is no big circle (or DQPT) in the former quench whereas the latter one possesses two big circles (or two types of DQPT). In addition, there is no accidental fixed point appearing in both of the quenches. The dynamical Chern numbers obtained by our experiment are -0.020 and 0.201 , respectively. It is clear that both of the quenches belong to the class (0a).

We also investigate the quenched SSQW starting from a SSQW in the topological phase $\left(+\frac{1}{2},+\frac{1}{2}\right)$ with $\theta_{1}=8.6 \pi / 9$, $\theta_{2}=\pi / 3$ and ending in a SSQW in another topologically nontrivial phase $\left(-\frac{1}{2},-\frac{1}{2}\right)$ with $\theta_{1}=-2 \pi / 3, \theta_{2}=\pi / 2$. Figure 8(a) shows the quenching strategy in a phase diagram. The time evolving spin states on the Bloch sphere for each quasimomentum $k$ are shown in (b) and the radius of the fitted circles are shown in (d). By observing the radius of the circles along the momentum, we can see that there is another fixed point $\left[r\left(k_{\mathrm{F}}\right)=0\right]$ appearing in $[0, \pi)$ [this quench belongs to subclass $(0 \mathrm{~b})]$. The fixed momentum $k_{\mathrm{F}}$ depends on the parameters of the quench; it is an accidental fixed point. In addition, this kind of fixed point may disappear after changing the time frame. At the same time, there are two big circles appearing for $k \in[0, \pi)$ [each submanifold has a big circle (one type of DQPT)]. The corresponding Berry curvature is shown in (c) and the measured dynamical Chern number is 0.171 . The appearance of the accidental fixed momentum and the big circle is consistent with the results in [22]. In fact, all the quenches between the two topologically nontrivial phases $\left(-\frac{1}{2},-\frac{1}{2}\right)$ and $\left(+\frac{1}{2},+\frac{1}{2}\right)$ belong to this class.

Discussion of the experimental errors. Here we mainly consider the influence of counting noise. In our experiment, the total coincidence count between the herald photon and the final up-conversion single photon is above $200 \mathrm{~Hz}$, and for each projecting basis the integral time is set to 200 seconds. Consequently, the total count for each trial of reconstructing the wave function is around 40000 . The error of the wavefunction fidelity is then estimated with Monte Carlo methods $[68,69]$. In this case, the error of fidelity induced by counting noise is typically $10^{-4}$ for the first step, and slowly grows bigger along with the time step of evolution. For the finial state at step 10, the value of error is estimated to be around $10^{-2}$. The degeneration is mainly induced by the decoherence as well as the increasing number of imperfect optical elements used in experiment. However, benefiting from the collinear architecture of optical interferometer, the fidelity of final states after ten full split-step QWs is still high enough to reconstruct the Berry curvature on the momentum-time manifold. As the Bloch vectors presented in panel (b) of Figs. 5, 6, and 8 are directly given by the Fourier transform of the wavefunction in site space, the errors for the time evolution vectors can been directly estimated by the corresponding errors of the wave-function fidelity.

While the fidelity of the Bloch vectors is high enough to reconstruct the time evolution circles and the corresponding Berry curvatures, the divergence of the Bloch vectors $\hat{\boldsymbol{n}}_{k}(t)$ for every quasimomentum $k$ does affect the accuracy of fitted circles. We can estimate the divergence by calculating the standard variance of $\left\{\left|\hat{\boldsymbol{n}}_{k}(t)-\hat{\boldsymbol{n}}_{k}^{f}\right|\right\}$ with $t=1, \ldots, 11$. The corresponding standard errors are presented in panel (d) of Figs. 5, 6, and 8. How well the circle fitting proceeds can also be estimated via hypothesis testing $[68,69]$. Here, we have four parameters to determine the time evolution circle of quasimomentum $k$, i.e., the radius $r(k)$ and a normalized vector $\hat{\boldsymbol{n}}_{k}^{f}$ giving the normal of the plane in which the circle is located. The sampling size for each circle is 11 , which is determined by the total number of steps; consequently it reads 11. The number of unconstrained variables, known as the the number of degrees of freedom, is then given with $v=7$. For Fig. 5, the estimated $\chi_{v}^{2}$ ranges from 0.2 to 4.5 , which indicates a high quality of our fitted circle.

The Berry curvature is defined as $\left[\hat{\boldsymbol{n}}_{k}(t) \times \partial_{t^{\prime}} \hat{\boldsymbol{n}}_{k}(t)\right]$. $\partial_{k} \hat{\boldsymbol{n}}_{k}(t)$, i.e., the integral kernel in Eq. (7). The differential terms result in the local value of Berry phase being particularly sensitive to the noise of the Bloch vector $\hat{\boldsymbol{n}}_{k}(t)$. The experimentally reconstructed Berry curvatures exhibit local minima and maxima, and even significantly diverge from 
(a)

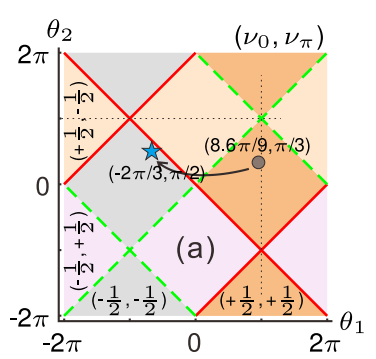

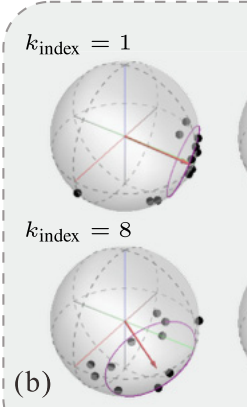
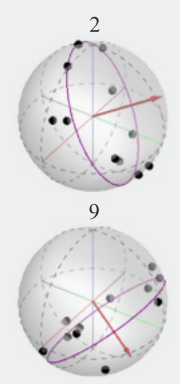
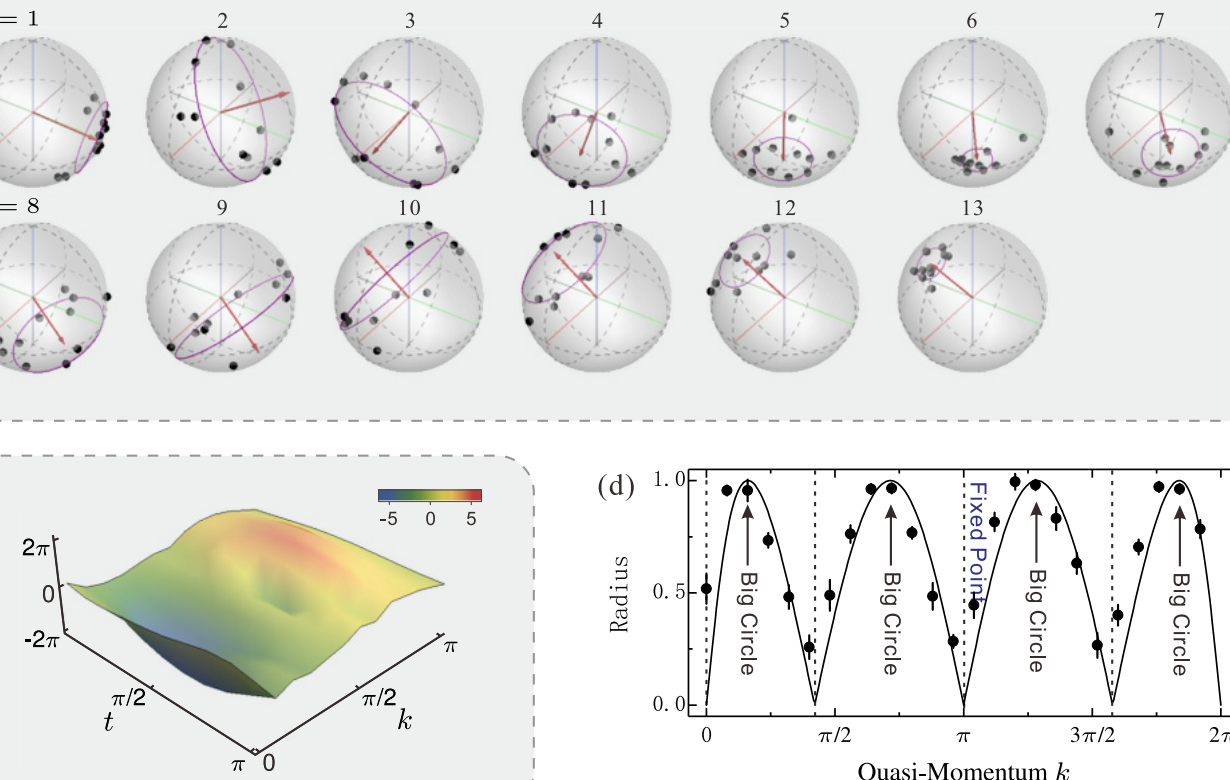

(d)
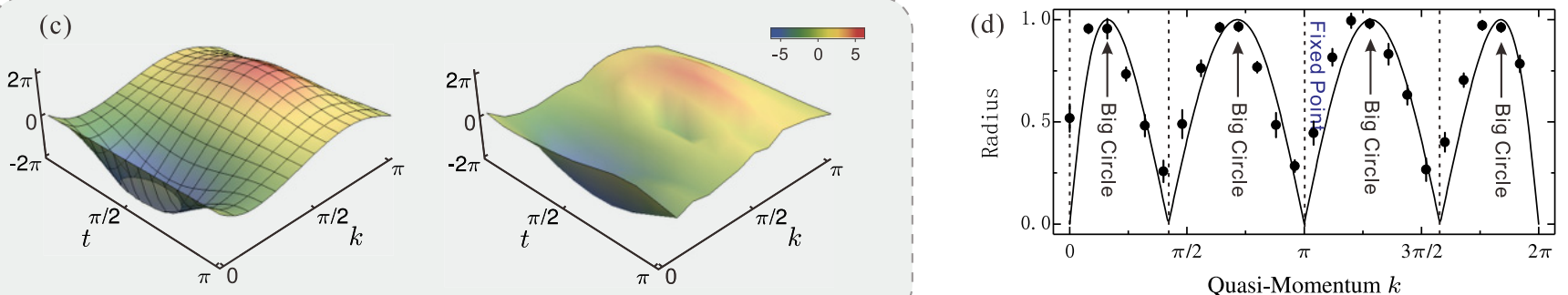

FIG. 8. Experimental results for a quantum quench between different topological (nonequivalent) nontrivial phases. (a) Quenching strategy in a phase diagram. We start from the flat band in nontrivial phase $\left(+\frac{1}{2},+\frac{1}{2}\right)$ with $\theta_{1}=\pi$. The system is then initialized in the ground state of a Hamiltonian with $\theta_{1}=8.6 \pi / 9, \theta_{2}=\pi / 3$ after performing three steps of adiabatic evolution. The quench ends in the nonequivalent topological phase $\left(-\frac{1}{2},-\frac{1}{2}\right)$ with $\theta_{1}=-2 \pi / 3, \theta_{2}=\pi / 2$. (b) Time evolution of the Bloch vectors for different quasimomenta $k$ (indexed by the number on the top of each Bloch sphere) shown as points. The circles on the sphere are fitted from the states indicating the time evolution trajectories. The red vectors indicate the direction of the eigenstate of the final Hamiltonian. (c) Corresponding Berry curvature in the momentum-time manifold, where the left panel is for the theory and the right panel is for the experiment. (d) Radius of the time evolution circle as a function of quasimomentum $k$. The points denote experimental results and the solid line denotes the theoretical prediction. The error bars represent the standard errors.

theoretical predictions for some local positions. However, as a global invariant defined on the whole Berry curvature, the dynamical Chern number is not extensively affected. As our platform does not sample the momentum space continuously and we cannot exactly access the quasimomentum $k=\pi$, the final dynamical Chern numbers found in experiments have a systematic deviation.

\section{CONCLUSION}

In summary, we have experimentally investigated the dynamical topology hidden in a periodically driven system. The quantum quenches are carried out on our photonic QW platform, which has the ability of reconstructing the full wave function of a single-photon walker in large scale. We experimentally rebuilt the time evolution trajectory and the corresponding Berry curvature in momentum-time space. According to the experimentally measured dynamical Chern numbers, which are defined on the momentum-time manifold, the quenched QWs can be completely classified into different classes. Furthermore, our results show that the classes of the quenches are related closely to the relevant quasi-equilibrium topological invariants.

We further found that the number of types of DQPT, which can also be used in the classification of quantum quenches via another dynamical topological invariant, DTOP, is one to one, corresponding to the number of big circles on the momentumtime manifold. While both of the integer-quantized numbers, i.e., DTOP and dynamical Chern number, can be used to classify the quantum quench, our results clearly show they are totally different: DTOP is related to the big circles and dynamical Chern number to the fixed points. Since the complete classification by dynamical Chern number is only dependent on the quasiequilibrium bulk invariants, it is more suitable to be used in such a task.

While the dynamical Chern number and the visual tool of the time evolution circle are defined specifically in two-level spin systems, our experimental findings in the rising topic of quantum nonequilibrium physics, which are of great interest to the community, will certainly inspire further studies especially in multilevel and multidimensional systems. In addition, for clarifying the relations among the system symmetries, the fixed points and the DQPTs will be extremely helpful in further works.

\section{ACKNOWLEDGMENTS}

This work was supported by National Key Research and Development Program of China (Grants No. 2017YFA0304100, No. 2016YFA0302700), the National Natural Science Foundation of China (Grants No. 11874343, No. 61805228, No. 11774335, No. 11821404, No. 61725504), the Key Research Program of Frontier Sciences, CAS (Grants No. QYZDY-SSW-SLH003), the Science Foundation of the CAS (Grants No. ZDRW-XH-2019-1), the Fundamental Research Funds for the Central Universities (Grants No. WK2470000026), the National Postdoctoral Program for Innovative Talents (Grants No. BX201600146), 
the China Postdoctoral Science Foundation (Grants No. 2017M612073), and the Anhui Initiative in Quantum Information Technologies (Grants No. AHY020100, No. AHY060300).

\section{APPENDIX A: APPEARANCE OF BIG CIRCLES}

According to the formula in Eq. (5) in the main text, the radius of the orbit of the time-dependent state with a quasimomentum $k$ can be calculated as

$$
r=\sqrt{1-\left(\hat{\boldsymbol{n}}_{k}^{i} \cdot \hat{\boldsymbol{n}}_{k}^{f}\right)^{2}}
$$

where $\hat{\boldsymbol{n}}_{k}^{i}\left(\hat{\boldsymbol{n}}_{k}^{f}\right)$ is the Bloch vector corresponding to the ground state of the initial (final) effective Hamiltonian $H_{i}(k)$ $\left[H_{f}(k)\right]$.

Suppose the SSQW is performed in a given time frame, whose time evolution operator in one period is $U=$ $\sqrt{R\left(\theta_{1}\right)} S_{-} R\left(\theta_{2}\right) S_{+} \sqrt{R\left(\theta_{1}\right)}$; it is easy to get

$$
\hat{\boldsymbol{n}}_{k}^{i} \cdot \hat{\boldsymbol{n}}_{k}^{f}=\frac{a x^{2}+b x+c}{\left|\sin E_{k}^{i} \sin E_{k}^{f}\right|},
$$

where $x \equiv \cos (k)$ within the range $x \in[-1,1]$ and

$$
\begin{aligned}
& a=\sin \left(\theta_{1 i} / 2\right) \cos \left(\theta_{2 i} / 2\right) \sin \left(\theta_{1 f} / 2\right) \cos \left(\theta_{2 f} / 2\right)-\cos \left(\theta_{2 i} / 2\right) \cos \left(\theta_{2 f} / 2\right), \\
& b=\cos \left(\theta_{1 i} / 2\right) \sin \left(\theta_{2 i} / 2\right) \sin \left(\theta_{1 f} / 2\right) \cos \left(\theta_{2 f} / 2\right)+\sin \left(\theta_{1 i} / 2\right) \cos \left(\theta_{2 i} / 2\right) \cos \left(\theta_{1 f} / 2\right) \sin \left(\theta_{2 f} / 2\right), \\
& c=\cos \left(\theta_{1 i} / 2\right) \sin \left(\theta_{2 i} / 2\right) \cos \left(\theta_{1 f} / 2\right) \sin \left(\theta_{2 f} / 2\right)+\cos \left(\theta_{2 i} / 2\right) \cos \left(\theta_{2 f} / 2\right) .
\end{aligned}
$$

Obviously, if the radius is equal to $1, \hat{\boldsymbol{n}}_{k}^{i} \cdot \hat{\boldsymbol{n}}_{k}^{f}$ should be equal to 0 , which means that $f(x)=a x^{2}+b x+c$ should have zero points for $x \in[-1,1]$. Now, we consider the property of the quadratic function $f(x)$ in that interval. First, the following relation can be obtained directly:

$$
\begin{aligned}
f(1) & =\sin \left[\left(\theta_{1 i}+\theta_{2 i}\right) / 2\right] \sin \left[\left(\theta_{1 f}+\theta_{2 f}\right) / 2\right], \\
f(-1) & =\sin \left[\left(\theta_{1 i}-\theta_{2 i}\right) / 2\right] \sin \left[\left(\theta_{1 f}-\theta_{2 f}\right) / 2\right] .
\end{aligned}
$$

To investigate the number of zeros between -1 and 1 , we need to consider the sign of $f(1) \times f(-1)$ such that if $f(1) \times$ $f(-1)>0$ there are an even number of zeros, otherwise if $f(1) \times f(-1)<0$ there are an odd number of zeros. Before considering the sign of $f(1) \times f(-1)$, first we investigate the sign of the function

$$
\begin{aligned}
u\left(\theta_{1}, \theta_{2}\right) & =\sin \left[\left(\theta_{1}+\theta_{2}\right) / 2\right] \sin \left[\left(\theta_{1}-\theta_{2}\right) / 2\right] \\
& =\sin ^{2}\left[\theta_{1} / 2\right] \cos ^{2}\left[\theta_{2} / 2\right]-\cos ^{2}\left[\theta_{1} / 2\right] \sin ^{2}\left[\theta_{2} / 2\right]:
\end{aligned}
$$

(1) If the SSQW with the parameter $\left(\theta_{1}, \theta_{2}\right)$ is located in the trivial phase, that is,

$$
\left|\tan \left[\theta_{2} / 2\right] / \tan \left[\theta_{1} / 2\right]\right|>1,
$$

we have $u\left(\theta_{1}, \theta_{2}\right)<0$.

(2) If the SSQW with the parameter $\left(\theta_{1}, \theta_{2}\right)$ is located in the nontrivial phase, that is,

$$
\left|\tan \left[\theta_{2} / 2\right] / \tan \left[\theta_{1} / 2\right]\right|<1,
$$

we have $u\left(\theta_{1}, \theta_{2}\right)>0$.
Now, we consider the relation between the number of the big circles appearing in the quenches and the topologies of the initial and final SSQWs.

\section{Quenched SSQW between topologically trivial and nontrivial phases}

In this case, $f(1) \times f(-1)<0$, meaning $f(x)$ has odd zeros in the interval $x \in[-1,1]$. Since $f(x)$ is a quadratic function with only two zeros at most, it can only have one zero in the interval $x \in[-1,1]$, i.e., only one big circle exists.

\section{Quenched SSQW between topologically (nonequivalent) nontrivial phases}

For quenches between two nontrivial phases, we have $f(1) \times f(-1)>0$, meaning $f(x)$ has an even number of zeros in the interval $x \in[-1,1]$. Therefore, $f(x)$ may have two zeros or have no zeros in that region. To determine the exact number of zeros, first we calculate the discriminant of $f(x)$. If the function $f(x)$ has zeros in that interval, the discriminant $\Delta$ should be larger than 0 .

For convenience, we do not calculate $\Delta$ directly. Instead, we calculate the function $g$ obtained by dividing $\Delta$ with some positive number, that is,

$$
\begin{aligned}
g & =\frac{b^{2}-4 a c}{\left[\cos \left(\theta_{1 i} / 2\right) \cos \left(\theta_{2 i} / 2\right) \cos \left(\theta_{1 f} / 2\right) \cos \left(\theta_{2 f} / 2\right)\right]^{2}} \\
& =a_{1} \tan ^{2}\left[\theta_{2 f} / 2\right]+b_{1} \tan \left[\theta_{2 f} / 2\right]+c_{1},
\end{aligned}
$$

where

$$
\begin{aligned}
& a_{1}=\tan ^{2}\left[\theta_{1 i} / 2\right], \\
& b_{1}=4 \sec \left[\theta_{1 i} / 2\right] \sec \left[\theta_{1 f} / 2\right] \tan \left[\theta_{2 i} / 2\right]-2 \tan \left[\theta_{1 i} / 2\right] \tan \left[\theta_{2 i} / 2\right] \tan \left[\theta_{1 f} / 2\right], \\
& c_{1}=4 \sec ^{2}\left[\theta_{1 i} / 2\right] \sec ^{2}\left[\theta_{1 f} / 2\right]+\tan ^{2}\left[\theta_{2 i} / 2\right] \tan ^{2}\left[\theta_{1 f} / 2\right]-4 \sec \left[\theta_{1 i} / 2\right] \sec \left[\theta_{1 f} / 2\right] \tan \left[\theta_{1 i} / 2\right] \tan \left[\theta_{1 f} / 2\right] .
\end{aligned}
$$

The function $g$ is viewed as a quadratic function of $\tan \left[\theta_{2 f} / 2\right]$, whose discriminant is

$$
b_{1}^{2}-4 a_{1} c_{1}=-16\left[\tan ^{2}\left(\theta_{1 i} / 2\right)-\tan ^{2}\left(\theta_{2 i} / 2\right)\right] \frac{1-\sin \left(\theta_{1 i} / 2\right) \sin \left(\theta_{1 f} / 2\right)}{\cos ^{2}\left(\theta_{1 i} / 2\right) \cos ^{2}\left(\theta_{1 f} / 2\right)} .
$$


In our current quench, the initial SSQW is in the nontrivial topological phase and we can obtain $\tan ^{2}\left(\theta_{1 i} / 2\right)-$ $\tan ^{2}\left(\theta_{2 i} / 2\right)>0$, thus $b_{1}^{2}-4 a_{1} c_{1}<0$. In addition, $a_{1}=$ $\tan ^{2}\left(\theta_{1 i} / 2\right)>0$. Consequently, we can conclude that the function $g$ is always positive. In other words, the discriminant of $f(x)$, i.e., $b^{2}-4 a c$, is positive for all quenches from the nontrivial topological phases.

A positive value for the discriminant of $f(x)$ means that the function has two real zeros (denoted as $x_{1}$ and $x_{2}$ ). Then, the question would be how many zeros exist in the interval $x \in[-1,1]$, i.e., 0 or 2 . We can determine the number of zeros by calculating the product of the zeros: if there are two zeros, their product $\left|x_{1} x_{2}\right|=|c / a|$ should be smaller than 1 ; otherwise, if there are no zeros, their product $\left|x_{1} x_{2}\right|=|c / a|$ should be larger than 1 .

(1) In a quench ending in the equivalent nontrivial phases, we have $1-\frac{\tan \left[\theta_{2 i} / 2\right] \tan \left[\theta_{2 f} / 2\right]}{\tan \left[\theta_{1 i} / 2\right] \tan \left[\theta_{1 f} / 2\right]}<2<\frac{2}{\sin \left[\theta_{1 i} / 2\right] \sin \left[\theta_{1 f} / 2\right]}$; then by multiplying $1+\frac{\tan \left[\theta_{2 i} / 2\right] \tan \left[\theta_{2 f} / 2\right]}{\tan \left[\theta_{1 i} / 2\right] \tan \left[\theta_{1 f} / 2\right]}$ to both sides at the same time, we can easily derive $\left|\frac{c}{a}\right|>1$, which means that there are no zeros.

(2) In a quench ending in a nonequivalent nontrivial phase, we have $\frac{2}{\sin \left[\theta_{1 i} / 2\right] \sin \left[\theta_{1 f} / 2\right]}<0<1-\frac{\tan \left[\theta_{2 i} / 2\right] \tan \left[\theta_{2 f} / 2\right]}{\tan \left[\theta_{1 i} / 2\right] \tan \left[\theta_{1 f} / 2\right]}$; similarly, we can get $\left|\frac{c}{a}\right|<1$, which means that there are two zeros.

Consequently, we can conclude that there is no big circle appearing in $k \in[0, \pi)$ when quenching the SSQW in the same topologically nontrivial phase and between equivalent topologically nontrivial phases, and there exist two big circles appearing in $k \in[0, \pi)$ when quenching the SSQW between nonequivalent topologically nontrivial phases.

\section{Quenched SSQW between trivial phases}

In this scenario, $f(1) \times f(-1)$ is positive and there may be no zeros or two zeros in the interval $x \in[-1,1]$. However, being different from the quenches starting from topologically nontrivial phases, this scenario is much more complicated, because an explicitly analytic conclusion from the quasiequilibrium picture cannot be reached.

However, we can prove that if the initial and final SSQW are in the same color region (from red to red, or from blue to blue; see Fig. 9), there is no big circle.

Suppose the initial and final SSQWs are in the same color region; we prove this claim by separating the region into two cases:

(a) If $\sin \left[\theta_{1 i} / 2\right] \times \sin \left[\theta_{1 f} / 2\right]>0$ (both of the initial and final SSQWs are on the same side of the purple line in Fig. 9), with the signatures we show in Fig. 9 and the condition of the initial and final SSQWs being in the same color region, we have $1-\frac{\tan \left[\theta_{2 i} / 2\right] \tan \left[\theta_{2 f} / 2\right]}{\tan \left[\theta_{1 i} / 2\right] \tan \left[\theta_{1 f} / 2\right]}<0<\frac{2}{\sin \left[\theta_{1 i} / 2\right] \sin \left[\theta_{1 f} / 2\right]}$ and $1+\frac{\tan \left[\theta_{2 i} / 2\right] \tan \left[\theta_{2 f} / 2\right]}{\tan \left[\theta_{1 i} / 2\right] \tan \left[\theta_{1 f} / 2\right]}>0$. By the same derivation as in Appendix A 2, we can get $\left|\frac{c}{a}\right|>1$, meaning that there is no big circle.

(b) If $\sin \left(\theta_{1 i} / 2\right) \times \sin \left(\theta_{1 f} / 2\right)<0$ (the initial and final SSQWs are on different sides of the purple line in Fig. 9), with the signatures we show in Fig. 9 and the condition of the initial and final SSQWs being in the same color region, we can obtain $1-\frac{\tan \left[\theta_{2 i} / 2\right] \tan \left[\theta_{2 f} / 2\right]}{\tan \left[\theta_{1 i} / 2\right] \tan \left[\theta_{1 f} / 2\right]}>0>\frac{2}{\sin \left[\theta_{1 i} / 2\right] \sin \left[\theta_{1 f} / 2\right]}$ and

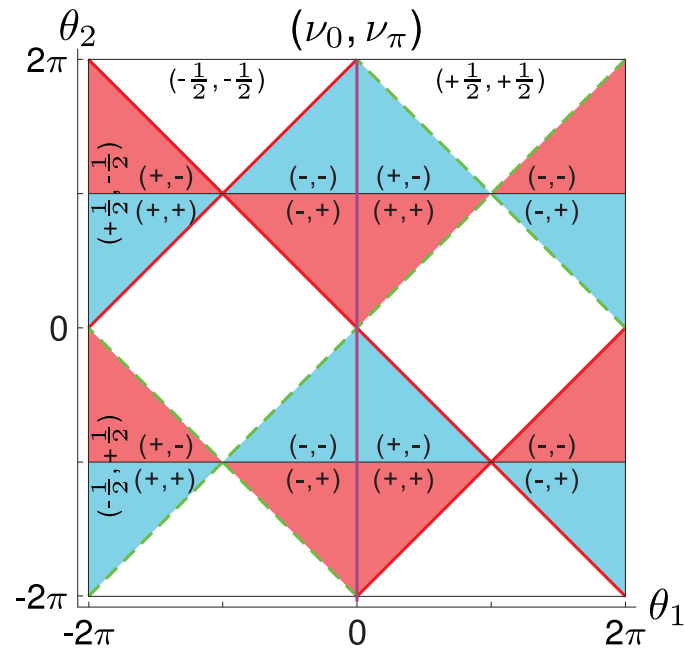

FIG. 9. To consider the big circle and accidental fixed points appearing in the quenches between the SSQWs in topologically trivial phases, each trivial phase in the quasiequilibrium phase diagram is further divided into regions: one is colored red and the other is colored blue. The signatures in the bracket in each region correspond to the signatures of $\left(\tan \left[\theta_{1} / 2\right], \tan \left[\theta_{2} / 2\right]\right)$ in each region. The green line represents the line $\theta_{1}=0$.

$1+\frac{\tan \left[\theta_{2 i} / 2\right] \tan \left[\theta_{2 f} / 2\right]}{\tan \left[\theta_{1 i} / 2\right] \tan \left[\theta_{1 f} / 2\right]}<0$. By the same derivation as in subsection $b$, we can get $\left|\frac{c}{a}\right|>1$, which means that there is no big circle.

Thus, we can conclude that there is no big circle when the initial and final SSQWs are in the same color region.

Otherwise, as discussed in the result related to Fig. 7, if we start the quenches from the red point in one trivial phase, numerical results show that, besides most regions where no big circle appears, regions exist also (purple in the figure) where two big circles appear.

\section{Special case: $a=0$}

If $a=0$, the function $f(x)$ reduces to a linear function, which can happen in different scenarios: (1) $\theta_{1 i}=\theta_{1 f}= \pm \pi$, (2) $\theta_{2 i}= \pm \pi$, or (3) $\theta_{2 f}= \pm \pi$. For case (1), the quench can only happen in the same nontrivial phases. For cases (2) and (3), the quench is not strongly limited, i.e., there can be quenches from trivial to nontrivial phases, quenches from nontrivial to trivial phases, or quenches from trivial to trivial phases. In all these cases, the condition $f(1) \times f(-1)>0$ means that $f(x)$ has no zero in the interval $x \in[-1,1]$ since $f(x)$ is a linear function, and vice versa. Consequently, the previous conclusion obtained with $a \neq 0$ is still valid for this special case.

\section{Conclusion}

In conclusion, in the quenched SSQWs between two nonequivalent topologically nontrivial phases, two big circles will appear inside $k \in[0, \pi)$. In quenched SSQWs between trivial and nontrivial phases, there is only one big circle. In quenched SSQWs inside the same topologically nontrivial phases and between two equivalent topologically nontrivial phases, there is no big circle. In quenched SSQWs inside the 
same trivial phase or between different trivial phases, there may or may not exist two big circles.

\section{APPENDIX B: APPEARANCE OF FIXED POINTS}

As mentioned in the main text, the fixed points plays a key role in calculating the dynamical Chern number. There are two different types of fixed points: those determined by the symmetry and those emerging accidentally. As the former case has been well studied, here, we focus on accidental fixed points.

The fixed points can be revealed via parallel or antiparallel appearances between the initial and final Bloch vectors $\hat{\boldsymbol{n}}_{k}^{i}$ and $\hat{\boldsymbol{n}}^{f}$, respectively. In other words, if $k_{\mathrm{F}}$ is a fixed point in the quench, we have $R=\left|\hat{\boldsymbol{n}}_{k_{\mathrm{F}}}^{i} \times \hat{\boldsymbol{n}}_{k_{\mathrm{F}}}^{f}\right|=0$. To determine the fixed points in the quenches, we expand $R$ as

$$
\begin{aligned}
R= & \mid \frac{\left[\cos \left(\theta_{1 f} / 2\right) \sin \left(\theta_{2 f} / 2\right)+\sin \left(\theta_{1 f} / 2\right) \cos \left(\theta_{2 f} / 2\right) \cos k\right] \cos \left(\theta_{2 i} / 2\right) \sin k}{\sin \left(E_{k}^{i}\right) \sin \left(E_{k}^{f}\right)} \\
& -\frac{\left[\cos \left(\theta_{1 i} / 2\right) \sin \left(\theta_{2 i} / 2\right)+\sin \left(\theta_{1 i} / 2\right) \cos \left(\theta_{2 i} / 2\right) \cos k\right] \cos \left(\theta_{2 f} / 2\right) \sin k}{\sin \left(E_{k}^{i}\right) \sin \left(E_{k}^{f}\right)} \mid .
\end{aligned}
$$

To get the accidental fixed point $k_{\mathrm{F}}$, we need to find the nontrivial zeros of $R$. They are easy to get: when

$$
\cos k=\frac{\cos \left(\theta_{1 f} / 2\right) \tan \left(\theta_{2 f} / 2\right)-\cos \left(\theta_{1 i} / 2\right) \tan \left(\theta_{2 i} / 2\right)}{\sin \left(\theta_{1 i} / 2\right)-\sin \left(\theta_{1 f} / 2\right)},
$$

we have $R=0$. Suppose $\alpha$ takes the value of the right-hand and $|\alpha|<1$; obviously there is a fixed point $k_{\mathrm{F}}=\arccos \alpha$. It is easy to check that

$$
\begin{aligned}
|\alpha|<1 \Longleftrightarrow & \frac{1}{\sin \left[\theta_{1 i} / 2\right]^{2}}\left(\frac{\tan \left[\theta_{2 f} / 2\right]^{2}}{\tan \left[\theta_{1 f} / 2\right]^{2}}-1\right)+\frac{1}{\sin \left[\theta_{1 f} / 2\right]^{2}}\left(\frac{\tan \left[\theta_{2 i} / 2\right]^{2}}{\tan \left[\theta_{1 i} / 2\right]^{2}}-1\right) \\
& +\frac{2}{\sin \left[\theta_{1 i} / 2\right] \sin \left[\theta_{1 f} / 2\right]}\left(1-\frac{\tan \left[\theta_{2 i} / 2\right] \tan \left[\theta_{2 f} / 2\right]}{\tan \left[\theta_{1 i} / 2\right] \tan \left[\theta_{1 f} / 2\right]}\right)<0 .
\end{aligned}
$$

\section{Accidental fixed points in quenches between SSQWs} in two nonequivalent topologically nontrivial phases

In the following, we will prove that if the quenched SSQWs are between two nonequivalent topologically nontrivial phases, an additional accidental fixed point always occurs. For this kind of quenched SSQWs, $\sin \left(\theta_{1 i} / 2\right) \sin \left(\theta_{1 f} / 2\right)<0$ is always satisfied, hence, using Eq. (B2) and the property of the SSQWs in nontrivial phases, it is clear that $|\alpha|<1$. Therefore, the accidental fixed point must exist and its value is dependent on the parameters driving the quench.

\section{Accidental fixed points in quenches between SSQWs in topologically trivial phases}

As mentioned before, in quenches between two SSQWs in (the same or different) trivial phases, regions exist where two big circles appear. We can prove that having two big circles and an accidental fixed point cannot occur simultaneously.

First, we prove that if the initial and final SSQWs are in different color regions (from red to blue or from blue to red in Fig. 9), the accidental fixed point does not exist. Suppose the initial and final SSQWs are in different color regions; we prove this claim by separating the region into two cases:

(a) If $\sin \left(\theta_{1 i} / 2\right) \sin \left(\theta_{1 f} / 2\right)>0$ (both of the initial and final SSQWs are on the same side of the purple line in Fig. 9), with the signatures we show in Fig. 9 and from Eq. (B2), it is clear that $|\alpha|>1$, so there is no accidental fixed point for this case.

(b) If $\sin \left(\theta_{1 i} / 2\right) \sin \left(\theta_{1 f} / 2\right)<0$ (the initial and final SSQWs are on different sides of the purple line in Fig. 9), similarly, it is easy to check that $|\alpha|>1$ is still valid, so there is no accidental fixed point for this case as well.

From above, we can conclude that there is no accidental fixed point appearing when the initial and final SSQWs are in different color regions. Combining this with the conclusions we get in Appendix A 3, we can conclude that two big circles and an accidental fixed point cannot both happen.

Note that this proof does not preclude the existence of accidental fixed points in other quenched SSQWs; see Table I for details. As Fig. 3 shows, the circle point is the initial SSQW in the topologically nontrivial phase, and, if the final SSQW is in the purple shadowed area, the accident fixed point

TABLE I. Comparison of the classification of dynamical Chern number and big circles.

\begin{tabular}{lccc}
\hline \hline & Big circle & Accidental fixed point & Dynamical Chern number \\
\hline trivial $\leftrightarrow$ nontrivial & 1 & 1 or 0 & 1 \\
$\left( \pm \frac{1}{2}, \pm \frac{1}{2}\right) \rightarrow\left( \pm \frac{1}{2}, \pm \frac{1}{2}\right)$ & 0 & 0 or 1 & 0 \\
$\left(-\frac{1}{2},-\frac{1}{2}\right) \leftrightarrow\left(+\frac{1}{2},+\frac{1}{2}\right)$ & 2 & 1 & 0 \\
$\left( \pm \frac{1}{2}, \mp \frac{1}{2}\right) \rightarrow\left( \pm \frac{1}{2}, \mp \frac{1}{2}\right)$ & $2(0)$ & $0(0$ or 1$)$ & 0 \\
\hline \hline
\end{tabular}


will appear. It is easy to see that the shadowed area covers all different topologically nontrivial phases, partially covers the area in the same topologically nontrivial phase, and partially covers the area in topologically trivial phases.

\section{APPENDIX C: ACCIDENTAL FIXED POINT AND PERIODICAL DRIVING}

The accidental points depend on the parameters of the quenches and the time frames (exchanging the parameters $\theta_{1}$ and $\theta_{2}$ ). In fact, the appearance of the fixed point strongly depends on the starting time of the periodical driving; i.e., at certain starting time, an accidental fixed point will not appear. To demonstrate this point, we consider the SSQWs with Floquet operator $\left.U=U_{-} R\left(\theta_{2}\right) U_{+} R_{(} \theta_{1}\right)$, where $\hat{\boldsymbol{n}}_{k}^{i} \times \hat{\boldsymbol{n}}_{k}^{f}$ can be written as

$$
\begin{aligned}
\left(\hat{\boldsymbol{n}}_{k}^{i} \times \hat{\boldsymbol{n}}_{k}^{f}\right)_{x} & =\frac{-\sin k\left(\cos k \cos \left[\theta_{2 i} / 2\right] \cos \left[\theta_{2 f} / 2\right] \sin \left[\left(\theta_{1 i}-\theta_{1 f}\right) / 2\right]+\cos \left[\theta_{1 i} / 2\right] \cos \left[\theta_{1 f} / 2\right] \sin \left[\left(\theta_{2 i}-\theta_{2 f}\right) / 2\right]\right.}{\sin E_{k}^{i} \sin E_{k}^{f}} \\
\left(\hat{\boldsymbol{n}}_{k}^{i} \times \hat{\boldsymbol{n}}_{k}^{f}\right)_{y} & =\frac{\sin [k]^{2} \cos \left[\theta_{2 i} / 2\right] \cos \left[\theta_{2 f} / 2\right] \sin \left[\left(\theta_{1 i}-\theta_{1 f}\right) / 2\right]}{\sin E_{k}^{i} \sin E_{k}^{f}} \\
\left(\hat{\boldsymbol{n}}_{k}^{i} \times \hat{\boldsymbol{n}}_{k}^{f}\right)_{z} & =\frac{\sin k\left(\sin \left[\theta_{1 i} / 2\right] \cos \left[\theta_{2 i} / 2\right] \cos \left[\theta_{1 f} / 2\right] \sin \left[\theta_{2 f} / 2\right]-\cos \left[\theta_{1 i} / 2\right] \sin \left[\theta_{2 i} / 2\right] \sin \left[\theta_{1 f} / 2\right] \cos \left[\theta_{2 f} / 2\right]\right)}{\sin E_{k}^{i} \sin E_{k}^{f}} .
\end{aligned}
$$

Hence, the fixed points of these quenches can be obtained by finding the common zeros of the above three functions. A solution exists only when $k=0, \pi, 2 \pi$, which were determined by the particle-hole symmetry, and all the accidental fixed points disappear.

[1] J. G. Bednorz and K. A. Müller, Possible high $T_{c}$ superconductivity in the Ba-La-Cu-O system, Z. Phys. B 64, 189 (1986).

[2] D. C. Tsui, H. L. Stormer, and A. C. Gossard, Two-Dimensional Magnetotransport in the Extreme Quantum Limit, Phys. Rev. Lett. 48, 1559 (1982).

[3] R. B. Laughlin, Anomalous Quantum Hall Effect: An Incompressible Quantum Fluid with Fractionally Charged Excitations, Phys. Rev. Lett. 50, 1395 (1983).

[4] R. Jackiw and C. Rebbi, Solitons with fermion number $1 / 2$, Phys. Rev. D 13, 3398 (1976).

[5] Z. Wang, Y. Chong, J. D. Joannopoulos, and M. Soljaǒić, Observation of unidirectional backscattering-immune topological electromagnetic states, Nature (London) 461, 772 (2009).

[6] L. Lu, J. D. Joannopoulos, and M. Soljaǒić, Topological states in photonic systems, Nat. Phys. 12, 626 (2016).

[7] A. Dauphin and N. Goldman, Extracting the Chern Number from the Dynamics of a Fermi Gas: Implementing a Quantum Hall Bar for Cold Atoms, Phys. Rev. Lett. 111, 135302 (2013).

[8] G. Jotzu, M. Messer, R. Desbuquois, M. Lebrat, T. Uehlinger, D. Greif, and T. Esslinger, Experimental realization of the topological Haldane model with ultracold fermions, Nature (London) 515, 237 (2014).

[9] N. Goldman, J. C. Budich, and P. Zoller, Topological quantum matter with ultracold gases in optical lattices, Nat. Phys. 12, 639 (2016).

[10] D. N. Sheng, Z. Y. Weng, L. Sheng, and F. D. M. Haldane, Quantum Spin-Hall Effect and Topologically Invariant Chern Numbers, Phys. Rev. Lett. 97, 036808 (2006).

[11] X. Chen, Z.-C. Gu, Z.-X. Liu, and X.-G. Wen, Symmetryprotected topological orders in interacting bosonic systems, Science 338, 1604 (2012).

[12] M. Z. Hasan and C. L. Kane, Colloquium: Topological insulators, Rev. Mod. Phys. 82, 3045 (2010).

[13] X.-L. Qi and S.-C. Zhang, Topological insulators and superconductors, Rev. Mod. Phys. 83, 1057 (2011).
[14] S.-Q. Shen, Topological Insulators: Dirac Equation in Condensed Matter, Springer Series in Solid-State Sciences, Vol. 187 (Springer, Heidelberg, 2012).

[15] J. Eisert, M. Friesdorf, and C. Gogolin, Quantum many-body systems out of equilibrium, Nat. Phys. 11, 124 (2015).

[16] H. Markus, Dynamical quantum phase transitions: A review, Rep. Prog. Phys. 81, 054001 (2018).

[17] P. Calabrese and J. Cardy, Evolution of entanglement entropy in one-dimensional systems, J. Stat. Mech. (2005) P04010.

[18] P. Calabrese and J. Cardy, Time Dependence of Correlation Functions Following a Quantum Quench, Phys. Rev. Lett. 96, 136801 (2006).

[19] F. H. L. Essler, S. Evangelisti, and M. Fagotti, Dynamical Correlations After a Quantum Quench, Phys. Rev. Lett. 109, 247206 (2012).

[20] J. C. Budich and M. Heyl, Dynamical topological order parameters far from equilibrium, Phys. Rev. B 93, 085416 (2016).

[21] C. Wang, P. Zhang, X. Chen, J. Yu, and H. Zhai, Scheme to Measure the Topological Number of a Chern Insulator from Quench Dynamics, Phys. Rev. Lett. 118, 185701 (2017).

[22] X.-Y. Xu, Q.-Q. Wang, M. Heyl, J. C. Budich, W.-W. Pan, Z. Chen, M. Jan, K. Sun, J.-S. Xu, Y.-J. Han, C.-F. Li, and G.-C. Guo, Measuring a dynamical topological order parameter in quantum walks, arXiv:1808.03930.

[23] M. Tarnowski, F. N. Ünal, N. Fläschner, A. E. Benno S. Rem, K. Sengstock, and C. Weitenberg, Characterizing topology by dynamics: Chern number from linking number, Nat. Commun. 10, 1728 (2019).

[24] N. Fläschner, D. Vogel, M. Tarnowski, B. S. Rem, D. S Lühmann, M. Heyl, J. C. Budich, L. Mathey, K. Sengstock, and C. Weitenberg, Observation of dynamical vortices after quenches in a system with topology, Nat. Phys. 14, 265 (2018).

[25] C. Yang, L. Li, and S. Chen, Dynamical topological invariant after a quantum quench, Phys. Rev. B 97, 060304(R) (2018). 
[26] T. Kitagawa, E. Berg, M. Rudner, and E. Demler, Topological characterization of periodically driven quantum systems, Phys. Rev. B 82, 235114 (2010).

[27] L. Jiang, T. Kitagawa, J. Alicea, A. R. Akhmerov, D. Pekker, G. Refael, J. I. Cirac, E. Demler, M. D. Lukin, and P. Zoller, Majorana Fermions in Equilibrium and in Driven Cold-Atom Quantum Wires, Phys. Rev. Lett. 106, 220402 (2011).

[28] M. S. Rudner, N. H. Lindner, E. Berg, and M. Levin, Anomalous Edge States and the Bulk-Edge Correspondence for Periodically Driven Two-Dimensional Systems, Phys. Rev. X 3, 031005 (2013).

[29] J. K. Asbóth, Symmetries, topological phases, and bound states in the one-dimensional quantum walk, Phys. Rev. B 86, 195414 (2012).

[30] J. K. Asbóth and H. Obuse, Bulk-boundary correspondence for chiral symmetric quantum walks, Phys. Rev. B 88, 121406(R) (2013).

[31] J. K. Asbóth, B. Tarasinski, and P. Delplace, Chiral symmetry and bulk-boundary correspondence in periodically driven onedimensional systems, Phys. Rev. B 90, 125143 (2014).

[32] H. Obuse and N. Kawakami, Topological phases and delocalization of quantum walks in random environments, Phys. Rev. B 84, 195139 (2011).

[33] H. Obuse, J. K. Asbóth, Y. Nishimura, and N. Kawakami, Unveiling hidden topological phases of a one-dimensional Hadamard quantum walk, Phys. Rev. B 92, 045424 (2015).

[34] D. Carpentier, P. Delplace, M. Fruchart, and K. Gawędzki, Topological Index for Periodically Driven Time-Reversal Invariant 2D Systems, Phys. Rev. Lett. 114, 106806 (2015).

[35] Y. Aharonov, L. Davidovich, and N. Zagury, Quantum random walks, Phys. Rev. A 48, 1687 (1993).

[36] R. Portugal, Quantum Walks and Search Algorithms (Springer, Berlin, 2013).

[37] A. M. Childs, Universal Computation by Quantum Walk, Phys. Rev. Lett. 102, 180501 (2009).

[38] A. M. Childs, D. Gosset, and Z. Webb, Universal computation by multiparticle quantum walk, Science 339, 791 (2013).

[39] T. Kitagawa, M. S. Rudner, E. Berg, and E. Demler, Exploring topological phases with quantum walks, Phys. Rev. A 82, 033429 (2010).

[40] T. Kitagawa, Topological phenomena in quantum walks: Elementary introduction to the physics of topological phases, Quantum Inf. Process. 11, 1107 (2012).

[41] P. M. Preiss, R. Ma, M. E. Tai, A. Lukin, M. Rispoli, P. Zupancic, Y. Lahini, R. Islam, and M. Greiner, Strongly correlated quantum walks in optical lattices, Science 347, 1229 (2015).

[42] Z. Yan, Y.-R. Zhang, M. Gong, Y. Wu, Y. Zheng, S. Li, C. Wang, F. Liang, J. Lin, Y. Xu, C. Guo, L. Sun, C.-Z. Peng, K. Xia, H. Deng, H. Rong, J. Q. You, F. Nori, H. Fan, X. Zhu, and J.-W. Pan, Strongly correlated quantum walks with a 12-qubit superconducting processor, Science 364, 753 (2019).

[43] J. Wang and K. Manouchehri, Physical Implementation of Quantum Walks (Springer, New York, 2014).

[44] H. B. Perets, Y. Lahini, F. Pozzi, M. Sorel, R. Morandotti, and Y. Silberberg, Realization of Quantum Walks with Negligible Decoherence in Waveguide Lattices, Phys. Rev. Lett. 100, 170506 (2008).

[45] M. A. Broome, A. Fedrizzi, B. P. Lanyon, I. Kassal, A. AspuruGuzik, and A. G. White, Discrete Single-Photon Quantum
Walks with Tunable Decoherence, Phys. Rev. Lett. 104, 153602 (2010).

[46] A. Peruzzo, M. Lobino, J. C. F. Matthews, N. Matsuda, A Politi, K. Poulios, X. Q. Zhou, Y. Lahini, N. Ismail, K. Worhoff, Y. Bromberg, Y. Silberberg, M. G. Thompson, and J. L. O'Brien, Quantum walks of correlated photons, Science 329, 1500 (2010).

[47] A. Schreiber, A. Gábris, P. P. Rohde, K. Laiho, M. Štefaňák, V. Potoček, C. Hamilton, I. Jex, and C. Silberhorn, A 2D quantum walk simulation of two-particle dynamics, Science 336, 55 (2012).

[48] A. Crespi, R. Osellame, R. Ramponi, V. Giovannetti, R. Fazio, L. Sansoni, F. De Nicola, F. Sciarrino, and P. Mataloni, Anderson localization of entangled photons in an integrated quantum walk, Nat. Photonics 7, 322 (2013).

[49] F. Cardano, F. Massa, H. Qassim, E. Karimi, S. Slussarenko, D. Paparo, C. de Lisio, F. Sciarrino, E. Santamato, R. W. Boyd, and L. Marrucci, Quantum walks and wavepacket dynamics on a lattice with twisted photons, Sci. Adv. 1, e1500087 (2015).

[50] X.-Y. Xu, Q.-Q. Wang, W.-W. Pan, K. Sun, J.-S. Xu, G. Chen, J.-S. Tang, M. Gong, Y.-J. Han, C.-F. Li, and G.-C. Guo, Measuring the Winding Number in a Large-Scale Chiral Quantum Walk, Phys. Rev. Lett. 120, 260501 (2018).

[51] Q.-Q. Wang, X.-Y. Xu, W.-W. Pan, K. Sun, J.-S. Xu, G. Chen, Y.-J. Han, C.-F. Li, and G.-C. Guo, Dynamic-disorder-induced enhancement of entanglement in photonic quantum walks, Optica 5, 1136 (2018).

[52] M. Heyl, A. Polkovnikov, and S. Kehrein, Dynamical Quantum Phase Transitions in the Transverse-Field Ising Model, Phys. Rev. Lett. 110, 135704 (2013).

[53] W. P. Su, J. R. Schrieffer, and A. J. Heeger, Solitons in Polyacetylene, Phys. Rev. Lett. 42, 1698 (1979).

[54] A. Y. Kitaev, Fault-tolerant quantum computation by anyons, Ann. Phys. (NY) 303, 2 (2003).

[55] S. Suzuki, J.-i. Inoue, and B. K. Chakrabarti, Quantum Ising Phases and Transitions in Transverse Ising Models, 2nd ed., Lecture Notes in Physics, Vol. 862 (Springer, Heidelberg, 2013).

[56] M. S. Rudner and L. S. Levitov, Topological Transition in a Non-Hermitian Quantum Walk, Phys. Rev. Lett. 102, 065703 (2009).

[57] B. Tarasinski, J. K. Asbóth, and J. P. Dahlhaus, Scattering theory of topological phases in discrete-time quantum walks, Phys. Rev. A 89, 042327 (2014).

[58] C. Cedzich, T. Geib, F. A. Grünbaum, C. Stahl, L. Velázquez, A. H. Werner, and R. F. Werner, The topological classification of one-dimensional symmetric quantum walks, Ann. Inst. Henri Poincare 19, 325 (2018).

[59] C. Cedzich, F. A. Grünbaum, C. Stahl, L. Velázquez, A. H Werner, and R. F. Werner, Bulk-edge correspondence of onedimensional quantum walks, J. Phys. A: Math. Theor. 49, 21LT01 (2016).

[60] V. V. Ramasesh, E. Flurin, M. Rudner, I. Siddiqi, and N. Y. Yao, Direct Probe of Topological Invariants Using Bloch Oscillating Quantum Walks, Phys. Rev. Lett. 118, 130501 (2017).

[61] T. Rakovszky, J. K. Asbóth, and A. Alberti, Detecting topological invariants in chiral symmetric insulators via losses, Phys. Rev. B 95, 201407(R) (2017). 
[62] F. Cardano, M. Maffei, F. Massa, B. Piccirillo, C. de Lisio, G. De Filippis, V. Cataudella, E. Santamato, and L. Marrucci, Statistical moments of quantum-walk dynamics reveal topological quantum transitions, Nat. Commun. 7, 11439 (2016).

[63] F. Cardano, A. D’Errico, A. Dauphin, M. Maffei, B. Piccirillo, C. de Lisio, G. De Filippis, V. Cataudella, E. Santamato, L. Marrucci, M. Lewenstein, and P. Massignan, Detection of Zak phases and topological invariants in a chiral quantum walk of twisted photons, Nat. Commun. 8, 15516 (2017).

[64] L. Xiao, X. Zhan, Z. H. Bian, K. K. Wang, X. Zhang, X. P. Wang, J. Li, K. Mochizuki, D. Kim, N. Kawakami, W. Yi, H. Obuse, B. C. Sanders, and P. Xue, Observation of topological edge states in parity-time-symmetric quantum walks, Nat. Phys. 13, 1117 (2017).

[65] X. Zhan, L. Xiao, Z. Bian, K. Wang, X. Qiu, B. C. Sanders, W. $\mathrm{Yi}$, and P. Xue, Detecting Topological Invariants in Nonunitary
Discrete-Time Quantum Walks, Phys. Rev. Lett. 119, 130501 (2017).

[66] S. Barkhofen, T. Nitsche, F. Elster, L. Lorz, A. Gábris, I. Jex, and C. Silberhorn, Measuring topological invariants in disordered discrete-time quantum walks, Phys. Rev. A 96, 033846 (2017).

[67] E. Flurin, V. V. Ramasesh, S. Hacohen-Gourgy, L. S. Martin, N. Y. Yao, and I. Siddiqi, Observing Topological Invariants Using Quantum Walks in Superconducting Circuits, Phys. Rev. X 7, 031023 (2017).

[68] P. R. Bevington and D. K. Robinson, Data Reduction and Error Analysis for the Physical Sciences, 3rd ed. (McGraw-Hill, Boston, 2003).

[69] I. Hughes and T. P. A. Hase, Measurements and their Uncertainties: A Practical Guide to Modern Error Analysis (Oxford University Press, Oxford, 2010). 